\title{
Fate of the Runner in Hit-and-run Collisions
}

\author{
Alexandre Emsenhuber (ii) and Erik Asphaug (i) \\ Lunar and Planetary Laboratory, University of Arizona, 1629 E. University Blvd., Tucson, AZ 85721, USA; emsenhuber@lpl.arizona.edu \\ Received 2018 November 15; revised 2019 February 8; accepted 2019 March 2; published 2019 April 19
}

\begin{abstract}
In similar-sized planetary collisions, a significant part of the impactor often misses the target and continues downrange. We follow the dynamical evolution of "runners" from giant impacts to determine their ultimate fate. Surprisingly, runners reimpact their target planets only about half of the time for realistic collisional and dynamical scenarios. Otherwise, they remain in orbit for tens of millions of years (the limit of our $N$-body calculations) and longer, or they sometimes collide with a different planet than the first one. When the runner does return to collide again with the same target planet, its impact velocity is mainly constrained by the outcome of the prior collision. Impact angle and orientation, however, are unconstrained by the prior collision.
\end{abstract}

Key words: planets and satellites: formation - planets and satellites: terrestrial planets

\section{Introduction}

The late stage of terrestrial planet formation is dominated by collisions at near-escaping velocities between similar-sized planetary bodies, aka giant impacts (Wetherill 1985; Kokubo $\&$ Ida 2002). But giant impacts are seldom efficient when it comes to accretion (Agnor \& Asphaug 2004); more often than not, a significant part of the smaller of the two (the impactor) "misses" the larger target. These "hit-and-run collisions" (HRCs; Asphaug et al. 2006) can result in multiple escaping remnants or a single "runner," the impactor stripped of its exterior materials (Marcus et al. 2009). As discussed below and in Asphaug et al. (2006) and Gabriel et al. (2019), the basic reason is that a portion of the impactor "misses" the target, in the sense that their projected pathways do not fully overlap. For example, consider two differentiated planets, each with a core that is half the planet's radius. If they impact at only $30^{\circ}$, more head-on than most impacts, their cores will fly right past one another.

The fate of the runner turns out to be a nuanced and important problem. Shock dissipation and momentum transfer to the target decrease the relative velocity of the runner compared to its pre-impact velocity, relative to the target. It is helpful (but overly simplistic) to think of the runner as "bouncing" off the target with some damping. Depending on incoming velocity, there is a substantial deflection of the impact plane and change in trajectory during the nonaccretionary close encounter.

The impactor always hits the target at faster than the mutual escape velocity, having nonzero original relative motion. However, the runner-the remnant impactor-emerges slower than the impact speed. Depending on dissipation, the emergence velocity can be either slower or faster than the escape velocity. When it emerges slower than the escape velocity, it remains gravitationally bound, and the result is one or more successive collisions separated by a day or more, in the case of graze-and-merge collisions (GMCs; Leinhardt et al. 2010). When the runner escapes the target, we call the result an HRC. Explored here is the case where the escaping runner, orbiting the Sun, returns to the target on a timescale of thousands to millions of years (Jackson \& Wyatt 2012), what we call a hit-and-run return collision (HRR).
Despite the great variety of giant impact outcomes and the known inefficiency of giant impact accretion, most planetary system formation models still rely on collisions being unconditionally treated as perfect mergers, with a few exceptions, e.g., Alexander \& Agnor (1998) and Chambers (2013). The underlying rationale for the assumption of a perfect merger is that even if a giant impact creates multiple bodies (a target and a runner), they will remain on crossing orbits and thus collide again at some point, resulting in total accretion in the end. This seems reasonable enough but warrants further investigation if there are other bodies to perturb the trajectories. In addition, including multiple collision fragments increases the computational requirements, making the simulation very expensive or untenable.

An improved model treating large collisions more realistically has been used in Bonsor et al. (2015), Carter et al. (2015), and Leinhardt et al. (2015) to model the intermediate stage of terrestrial planet formation; significantly, it was found that a more realistic treatment of collisions would, as expected, increase the compositional diversity of remainders. The realistic treatment of giant impacts is also significant for the formation and evolution of gas giants and their $\mathrm{H} / \mathrm{He}$ envelopes (Schlichting et al. 2015). Gas giants form while the nebula is still present, during the first 3-6 Myr (Haisch et al. 2001), whereas terrestrial planet formation finishes over the next 100-200 Myr (e.g., Agnor et al. 1999; Chambers 2001). Depending on the timing and the mass distribution of giant impact remnants, the resulting bombardment by unfinished remnants can cause the loss of a giant planet envelope (Inamdar \& Schlichting 2016).

Runners from HRCs are mostly composed of the projectile and have a lower mass than the projectile. In characteristic HRCs, the outcome is a projectile interior, stripped of its outer layers (Marcus et al. 2009). This outcome could be able to explain several features of the solar system, such as Mercury's anomalously high bulk density (Benz et al. 1988, 2007; Asphaug \& Reufer 2014; Chau et al. 2018) and removal of water from icy planetesimals (Burger et al. 2018). Material exchange between the target and impactor during an HRC is also able to provide a means for material equilibration, where surviving runners can have signatures closer to their previous targets if they survive until today. Also, in the case of disrupted runners, surviving fragments can be genetically related, part of 
one original body, a kind of early catastrophic disruption that can occur at a low relative velocity (Asphaug 2017).

The aim of this work is to properly determine the destination of the runner in HRCs. If it comes right back in a slow effective merger, then it might not be overall that different from a GMC. If the runner comes back on a much longer timescale and with randomized orientation, or does not come back at all, the consequences are, of course, different and important. We model several such events with a hydrodynamical code to obtain the realistic end state of HRCs and track the remnants until those collide again. In the case of recollision, we determine the parameter of the second event and its type and search for any correlation (or lack thereof) with the first collision. This will provide clues as to whether perfect accretion is a justifiable simplification to accretion codes and determine the time that is ultimately needed for accretion between a pair of planets to play out in orbit.

\section{Methods}

We adopt a coupled approach, where the initial collision is modeled with a hydrodynamical scheme that captures the shock process and tidal interactions. Once the bodies are separated enough so that neither plays a significant role anymore, the results are then transferred into an $N$-body code to obtain the dynamical evolution of the resulting bodies. The $N$-body evolution is performed many times for each initial collision, assuming different impact orientations to obtain a statistical description.

\subsection{Collision Stage}

Collisions are modeled using the smoothed particle hydrodynamics (SPH) technique using a code especially suited for large-scale collisions (Reufer et al. 2012; Asphaug \& Reufer 2014; Emsenhuber et al. 2018). SPH is a Lagrangian method with material represented by particles. Quantities are retrieved by performing a kernel interpolation and spatial derivatives by interpolation of the underlying quantity with the kernel derivative (see, e.g., Monaghan 1992; Rosswog 2009 for reviews). We use the M-ANEOS equation of state (Thompson \& Lauson 1972; Melosh 2007) to obtain the pressure $p(\rho, u)$ and other necessary quantities for the hydrodynamical equations. In addition, self-gravity is included by the mean of a hierarchical spatial tree (Barnes \& Hut 1986).

We model grazing collisions (HRCs and GMCs) and study the spatial mixing of particles to ascertain the effectiveness of successive giant impacts, especially on isotopic equilibration. This kind of collision accounts for the vast majority of giant impacts. For collisions where the runner is not reaccreted in a short time (a couple of days), we also determine the spatial deflections that follow from the collision, the post-trajectory of the runner versus the pre-trajectory of the projectile. Because of the complex mass distribution and states of rotation, it is sometimes not obvious until quite late in the evolution that the collision will be a GMC and not an HRC. In the case of studying HRRs where the runner escapes the target, the calculation of HRRs has to advance the runner as an independent planet for thousands or millions of years using dynamical methods as described below.

The initial bodies are obtained by evolving initial spheres with particles uniformly spaced and reference density from the equation of state under self-gravity and pressure forces, applying a damping term and constant entropy until hydrostatic equilibrium is reached. The bodies are not spinning. Resolution is selected so that a $1 M_{\oplus}$ body with a chondritic composition, that is, 30wt\% iron core and $70 \mathrm{wt} \%$ silicate mantle, is represented by 500,000 particles. Collision modeling is performed in the two-body center-of-mass frame, beginning with the bodies several radii away, as this allows for tidal deformation and spin-up prior to the contact. These effects can strongly influence the outcome, compared to starting out the targets at the time of contact.

Collisions are first evolved to $24 \mathrm{hr}$ after initial contact. At this point, we compute the resulting properties of each body, including their orbital parameters. It is not trivial to determine the final independent bodies (e.g., target, runner) at this relatively early time, because there are spurious effects due to the fast rotation of the runner that can cause a large fraction of the runner particles (those rotating toward the target) to appear energetically bound to the target even though the two bodies are escaping from one another. The search for post-collision independent bodies is therefore performed starting with a friends-of-friends search $(\mathrm{FoF})$, followed by a determination of gravitational binding. The FoF clumps are treated as a single superparticle during the latter stage. See the Appendix for details and the necessity of such an approach. The choice of ending the hydrodynamical simulation at $24 \mathrm{hr}$ is to ensure that the body properties have converged. In all simulations presented hereafter, the resulting properties do not change by more than a few percent after $18 \mathrm{hr}$ (an example for mass convergence is provided in the Appendix).

In order to ease the comparison with the parameters of the return collision, we introduce two new quantities, $v_{\text {dep }}$ and $\theta_{\text {dep }}$, which are the departing velocity and angle of the secondary remnants with respect to the largest at a distance that is the sum of their radii after the collision. These would be identical to the returning collision velocity and angle if energy and angular momentum were conserved. The presence of the Sun and planets makes the return collision nonconservative. In the case of HRCs, the escaping runner must be tracked for many orbits until its next encounter with the target; this can be a close encounter leading to the escape of the runner or a follow-on collision that we model by mapping the outcome of one hydrodynamical simulation into another.

\subsection{Dynamical Evolution}

We map the target and runner emerging from one collision, ignoring collision products smaller than the two primary remnants, into the Mercury $N$-body code (Chambers 1999). The resulting bodies are followed until a subsequent collision occurs, or up to $20 \mathrm{Myr}$. To obtain statistics of the possible outcomes, we adopt a Monte Carlo approach assuming a range of possible pre-impact orbits. We consider the target to be on a circular orbit at $1 \mathrm{au}$, and the pre-impact orbit of the impactor is computed according to Jackson et al. (2018). That methodology provides the orbit for a head-on collision, as in the top panel of Figure 1, with the angles $\phi$ and $\theta$ providing the direction of the relative velocity vector. In the case of an oblique impact, a modification must be incorporated. We will assume that the same angles represent the direction of the asymptote of the hyperbolic orbit. We also introduce a third angle, $\psi$, that represents a rotation about this direction.

Neglecting the distance perpendicular to the eccentricity vector introduces only a small error. The deviation from a 


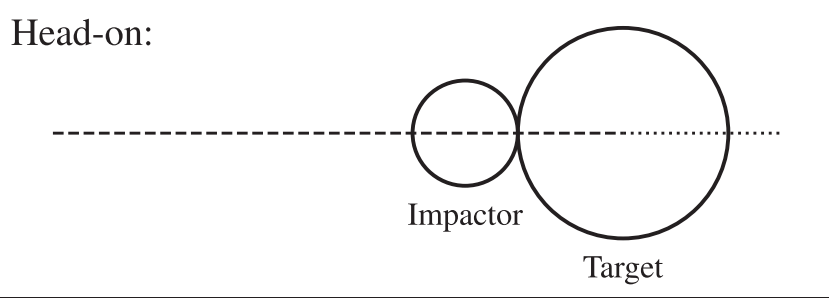

Oblique:

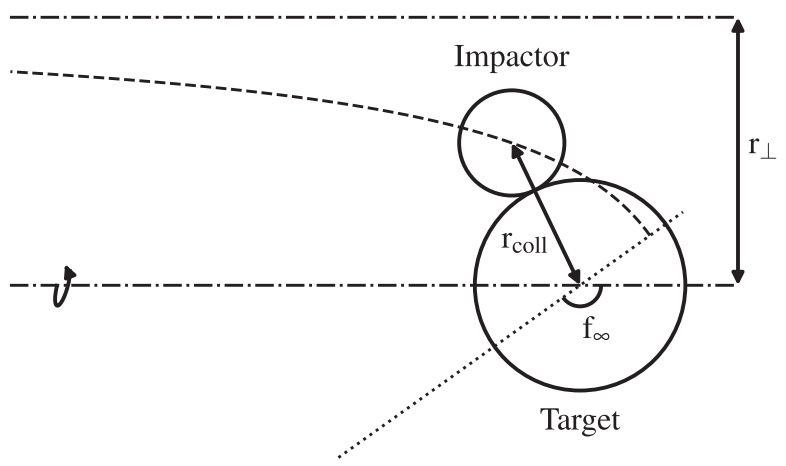

Figure 1. Sketch of the correction due to impact obliqueness. Note that a small mutual inclination of the orbits can cause a high inclination of the collisional plane. Top: case of a head-on collision, where the relative distance and velocity lie on the same axis. Bottom: oblique collision with $\theta_{\text {coll }}=45^{\circ}$ and $v_{\text {coll }} / v_{\text {esc }}=1.1$. The dashed lines represent the trajectory of the impactor, the dotted lines represent the major axis of the orbit, and the dash-dotted lines represent the asymptote of the hyperbola and its parallel that passes through the center of the target. At large separation, the relative position and velocity vectors tend to be parallel, with an offset $r_{\perp}$.

head-on orbit at large separation is given by

$$
\frac{r_{\perp}}{r_{\mathrm{coll}}}=\frac{v_{\mathrm{coll}} / v_{\mathrm{esc}}}{\sqrt{\left(v_{\mathrm{coll}} / v_{\mathrm{esc}}\right)^{2}-1}} \sin \theta_{\mathrm{coll}},
$$

with $r_{\text {coll }}=r_{\mathrm{tar}}+r_{\mathrm{imp}}$ the separation at initial contact (this relationship follows from energy and angular momentum conservation). If we assume two Earth-size planets colliding at $45^{\circ}$ with a velocity of $v_{\text {coll }} / v_{\text {esc }}=1.1$, then the offset at large separation is $r_{\perp} \approx 2.2 \times 10^{4} \mathrm{~km}$ or, compared to the semimajor axis, $r_{\perp} / a \approx 1.5 \times 10^{-4}$. This value would be the correction in eccentricity or inclination due to the obliqueness of the impact; it is sufficiently small compared to the encountered values of either quantity to be ignored. Higherimpact velocities lead to even smaller corrections.

For the majority of our modeling, we assume that $\phi$ and $\theta$ have an underlying uniform distribution in space, and $\psi$ also follows a uniform distribution. We also verify the robustness of the results against specific impact geometry by comparing to a set of collisions that happen only in the orbital plane. For this second series of initial conditions, we set $\theta=\pi / 2$ and $\psi=0$ while $\phi$ follows a uniform distribution. Our assumption that the pre-impact orbit of the target is circular should not lead to a singular situation during the dynamical evolution. As the target is also being deflected during the collision, its orbit will no longer be circular afterward.

Using one "snapshot" of a given original collision and rotating it in space to provide different dynamical initial states assumes that other planets or the Sun have negligible effects on the collision process. In ongoing work (Emsenhuber \& Asphaug 2019, in preparation), we do find that the GMCs of two satellites in the presence of a central planet are strongly affected by their orientation in space, but this happens because the Hill sphere is so small. The present situation is quite different. First, the Hill sphere of an Earth-mass object around the Sun is $\simeq 1 \%$ of its distance to the central star. At $1 \mathrm{au}$, the Hill sphere is $\simeq 1.5 \times 10^{6} \mathrm{~km}$, or more than 200 Earth radii. Only the most weakly bound GMCs would be affected by the presence of the Sun, and the region in the parameter space (impact velocity and angle) for which this happens is very narrow. To provide an idea of whether the transient remnant of the GMC might be affected by the presence of other bodies, the apocenter is computed for the period between the initial and second collision.

\subsection{Return Collision}

Dynamical evolution is used to provide the parameters of subsequent collisions in HRR scenarios, where the runner returns for a second giant impact. We do not perform direct SPH simulations of the returning collisions, however, as this would be a considerable resource requirement and unique in every case. Rather, we opt for ascertaining the return collision regime by using scaling laws, following either Leinhardt \& Stewart (2012) or Cambioni et al. (2019, hereafter C19) The former is a well-established model that is based on extensive hydrodynamical simulations. The latter case is an application of machine-learning supervised classification, where the underlying collisions were performed using a method and initial conditions similar to the present work.. In particular, the classes provided by the latter are consistent with the description of GMCs and HRCs used here.

\section{Results}

We describe the results of our numerical investigations, divided into the three sequential components of HRRs: the initial collisions, modeled using SPH; the dynamical evolution (with and without the presence of other planets), modeled using Mercury; and the return collisions, classified according to scaling laws. We search for correlations between initial and return collision parameters. Contrary to the assumptions made in previous research, for a large fraction of HRCs, there is no return collision, in which case we categorize the fate of the runner for up to 20 million yr, whether it impacts another planet (not the original target) or remains in orbit around the Sun.

\subsection{Initial Collisions}

We model a set of prior collisions where the mass of the target is always $m_{\mathrm{tar}}=0.9 M_{\oplus}$ and the impactor mass is either $m_{\mathrm{imp}}=0.2$ or $0.5 M_{\oplus}$, so that the mass ratio $\gamma=m_{\mathrm{imp}} / m_{\mathrm{tar}}$ is 0.22 and 0.56 , respectively. We limit our study to impact velocities in the range $v_{\text {coll }} / v_{\text {esc }}=1.1-1.2$, where $v_{\mathrm{esc}}=G\left(m_{\mathrm{tar}}+m_{\mathrm{imp}}\right) /\left(r_{\mathrm{tar}}+r_{\mathrm{imp}}\right)$ is the mutual escape velocity. The selected mass ratios fall within the expected range occurring during planetary formation. The smaller mass ratio is selected to be similar to potential moon-forming collisions (e.g., Reufer et al. 2012), whereas the higher one is close to the expected median of distribution during the overall formation process. More dissimilar bodies are also expected to lead to greater mass transfer from the impactor to the target during a collision, as equal-mass bodies should have none for symmetry. Different spins may break the symmetry, however. 

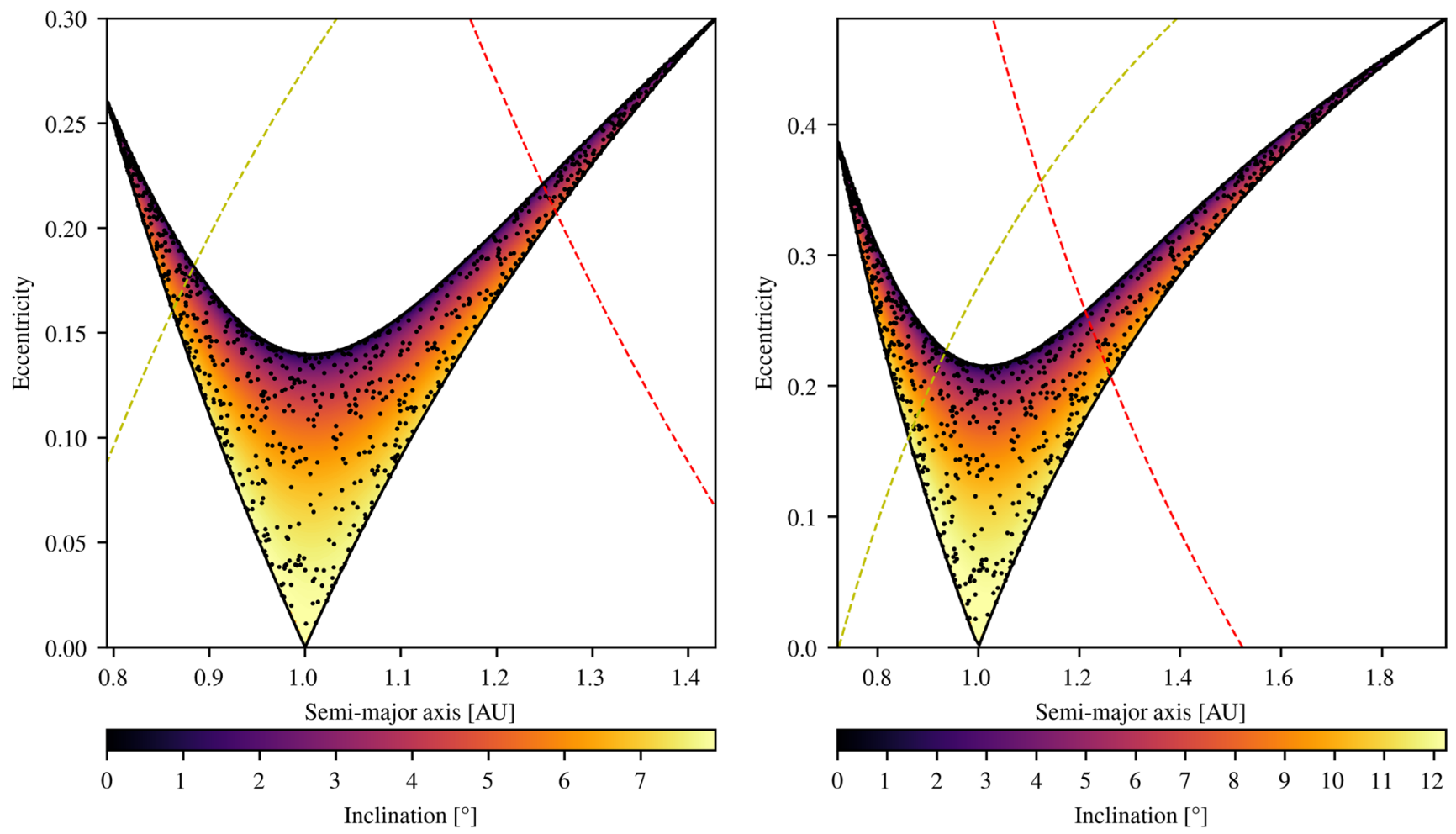

Figure 2. Pre-impact orbits of the impactors in our dynamical modeling. Left: case with $m_{\mathrm{imp}}=0.2 M_{\oplus}$ and $v_{\text {coll }} / v_{\mathrm{esc}}=1.10$. Right: $m_{\mathrm{imp}}=0.5 M_{\oplus}$ and $v_{\text {coll }} / v_{\text {esc }}=1.20$. These are the two extremes of our dynamical evolution; i.e., the former has the lowest relative velocity, while the latter has the highest. Orbits higher than the dashed yellow curve are Venus-crossing, and the ones higher than the dashed red curve are Mars-crossing.

Table 1

Initial and Final Conditions for the Prior Collision Simulations

\begin{tabular}{|c|c|c|c|c|c|c|c|c|c|c|c|c|c|c|}
\hline$m_{\mathrm{tar}}\left[M_{\oplus}\right]$ & $m_{\mathrm{imp}}\left[M_{\oplus}\right]$ & $\gamma$ & $\frac{v_{\text {coll }}}{v_{\text {esc }}}$ & $\theta_{\text {coll }}[\mathrm{deg}]$ & $m_{\mathrm{lr}}\left[M_{\oplus}\right]$ & $m_{\mathrm{sr}}\left[M_{\oplus}\right]$ & $\gamma_{\text {after }}$ & $m_{\text {lost }}\left[M_{\oplus}\right]$ & $\frac{\overline{v_{\text {dep }}}}{v_{\text {esc }}}$ & $\theta_{\text {dep }}[\mathrm{deg}]$ & $f_{\text {core }}^{1 \leftarrow i}$ & $f_{\text {core }}^{\mathrm{s} \leftarrow \mathrm{t}}$ & $f_{\operatorname{man}}^{\mathrm{l} \leftarrow \mathrm{i}}$ & $f_{\operatorname{man}}^{\mathrm{s} \leftarrow \mathrm{t}}$ \\
\hline & & & 1.10 & 55.0 & 0.92 & 0.18 & 0.19 & $8.0 \times 10^{-4}$ & 1.01 & 62 & $0.6 \%$ & $0.0 \%$ & $5.1 \%$ & $8.7 \%$ \\
\hline & & & 1.15 & 45.0 & $0.90^{\mathrm{a}}$ & $0.13^{\mathrm{a}}$ & $0.15^{\mathrm{a}}$ & $4.1 \times 10^{-3}$ & $0.98^{\mathrm{a}}$ & $53^{\mathrm{a}}$ & $4.1 \%$ & $0.0 \%$ & $4.7 \%$ & $14.2 \%$ \\
\hline & & & 1.15 & 52.5 & 0.92 & 0.18 & 0.19 & $1.4 \times 10^{-3}$ & 1.05 & 59 & $0.8 \%$ & $0.0 \%$ & $5.1 \%$ & $10.0 \%$ \\
\hline & & & 1.15 & 60.0 & 0.91 & 0.19 & 0.20 & $3.8 \times 10^{-4}$ & 1.08 & 63 & $0.0 \%$ & $0.0 \%$ & $3.3 \%$ & $5.7 \%$ \\
\hline & & & 1.20 & 42.5 & 0.95 & 0.14 & 0.14 & $7.2 \times 10^{-3}$ & 1.00 & 53 & $5.2 \%$ & $0.0 \%$ & $9.0 \%$ & $18.9 \%$ \\
\hline 0.9 & 0.5 & 0.56 & 1.10 & 45.0 & $1.38^{\mathrm{b}}$ & $\cdots$ & $\cdots$ & $1.6 \times 10^{-2}$ & $\ldots$ & $\ldots$ & $\cdots$ & $\ldots$ & $\ldots$ & $\cdots$ \\
\hline & & & 1.10 & 60.0 & 0.92 & 0.48 & 0.53 & $1.0 \times 10^{-3}$ & 1.00 & 60 & $0.0 \%$ & $0.0 \%$ & $6.1 \%$ & $6.9 \%$ \\
\hline & & & 1.15 & 45.0 & $0.87^{\mathrm{a}}$ & $0.44^{\mathrm{a}}$ & $0.50^{\mathrm{a}}$ & $4.3 \times 10^{-3}$ & $0.96^{\mathrm{a}}$ & $50^{\mathrm{a}}$ & $0.8 \%$ & $0.0 \%$ & $8.0 \%$ & $11.0 \%$ \\
\hline & & & 1.15 & 52.5 & 0.92 & 0.48 & 0.52 & $2.4 \times 10^{-3}$ & 1.03 & 56 & $0.0 \%$ & $0.0 \%$ & $8.0 \%$ & $10.2 \%$ \\
\hline & & & 1.15 & 60.0 & 0.91 & 0.49 & 0.54 & $1.1 \times 10^{-3}$ & 1.06 & 62 & $0.0 \%$ & $0.0 \%$ & $5.2 \%$ & $6.4 \%$ \\
\hline
\end{tabular}

Notes. See the main text for an explanation of the symbols.

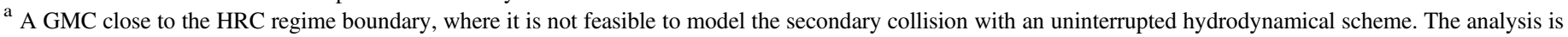
based on FoF rather than a gravity search.

${ }^{\mathrm{b}} \mathrm{A}$ GMC where the second collision can be modeled with an uninterrupted hydrodynamical scheme.

During the presence of the gas disk, our chosen velocity range is on the upper part of the distribution and close to the median found after the dispersal of the gas disk (Raymond et al. 2009; Stewart \& Leinhardt 2012). As for impact angle, we study collision angles greater than $40^{\circ}$ that result in grazing collisions (HRCs or GMCs) for these velocities. The corresponding pre-impact orbits of the impactors are shown in
Figure 2. The black dots represent each initial condition for the dynamical evolution. In the case of impacts happening in the orbital plane, all of the pre-impact orbits have $I=0$ and lie on the upper boundary of each plot. For the lowest-velocity case, $v_{\text {coll }} / v_{\text {esc }}=1.1$, this means that the impactor has an eccentricity of about 0.15 (if it has the same semimajor axis than the target, more otherwise), an inclination up to about $8^{\circ}$, 
Table 2

Additional Results for Initial Collisions That Are in the GMC but Where the Secondary Body Has Not Yet Been Accreted

\begin{tabular}{lcccccc}
\hline \hline$m_{\text {tar }}\left[M_{\oplus}\right]$ & $m_{\text {imp }}\left[M_{\oplus}\right]$ & $\gamma$ & $\frac{v_{\text {coll }}}{v_{\text {esc }}}$ & $\theta_{\text {coll }}[\mathrm{deg}]$ & $q / r_{\text {lr }}$ & $r_{\text {apo }}[\mathrm{km}]$ \\
\hline 0.9 & 0.2 & 0.22 & 1.10 & 52.5 & 1.22 & $5.87 \times 10^{5}$ \\
0.9 & 0.2 & 0.22 & 1.15 & 45.0 & 1.15 & $2.45 \times 10^{5}$ \\
0.9 & 0.5 & 0.56 & 1.15 & 45.0 & 1.15 & $1.74 \times 10^{5}$ \\
\hline
\end{tabular}

Note. The first five columns are the initial conditions. Here $q / r_{\mathrm{Ir}}$ is the pericenter, given in terms of the radius of the largest remnant; $r_{\text {apo }}$ is the apocenter; and $T$ is the orbital period.

or a combination of both. The pre-impact orbits of the larger impactors are slightly more eccentric or inclined for the same impact-to-escape velocity ratio, as the mutual escape velocity increases by nearly $7 \%$. The mass of the impactor more than doubles, but the separation at initial contact also increases, which counterbalances the effect.

The main results from the collision modeling are provided in Table 1. The first five columns provide the initial conditions, $m_{\mathrm{lr}}$ denotes the mass of the largest remnant, $m_{\mathrm{sr}}$ is the mass of the second remnant, $\gamma_{\text {after }}$ is the mass ratio between these two bodies, and $m_{\text {lost }}$ is the mass bound to neither the largest nor the second remnants. Here $v_{\mathrm{dep}} / v_{\mathrm{esc}}$ and $\theta_{\text {dep }}$ provide the orbital configuration of the second remnant with respect to the largest. The last four columns indicate material exchange occurring during the encounter, with the superscript $1 \leftarrow \mathrm{i}$ indicating the fraction of the largest remnant coming from the impactor and $\mathrm{s} \leftarrow \mathrm{t}$ indicating the fraction of the second remnant coming from the target. Some of these collisions are GMCs, indicated by either (1) the almost total accretion of the impactor, with no secondary remaining and only loose material, or (2) a bound secondary body that has not yet been accreted. In the latter case, we provide the results as found by the FoF search so that the bodies can be distinguished. The only exception to this is the lost mass, which still comes from the gravity search; so the masses shown on the table do not add up because bound material that is not part of an FoF body is not accounted for in any item. For these cases, we provide a few additional quantities in Table 2, which are computed assuming a twobody problem without external perturbation. The first quantity is the pericenter, given in terms of the radius of the larger body; the second is the apocenter; and the last one is the orbital period. For reference, the Hill radius of the Earth is roughly $1.5 \times 10^{6} \mathrm{~km}$, so that all of these transient bodies would remain within the Hill sphere during the orbit. What happens to a GMC when the apocenter is comparable to the Hill radius is the subject of ongoing research (A. Emsenhuber \& E. Asphaug 2019 , in preparation). A GMC with a return period of the order of 5 days could potentially be evolved with the hydrodynamical scheme with enough precision; longer-period GMCs would require huge computational resources for little benefit.

Some HRCs are just above the boundary of GMCs, meaning that the runner leaves the target with a velocity that is just above the mutual escape velocity. In terms of the first giant impact, the transition between GMCs and HRCs is smooth; the only way to discern the longest-period GMC from the lowestenergy HRC is to integrate the final bodies forward in time. As expected, the higher-velocity collisions at lower impact angles liberate greater quantities of loose material owing to the greater strength of the interaction. The exceptions are GMCs that involve multiple underlying encounters; for example, the GMC simulation with $m_{\mathrm{imp}}=0.5 M_{\oplus}, v_{\mathrm{coll}} / v_{\mathrm{esc}}=1.10, \theta_{\mathrm{coll}}=45^{\circ}$ has a much higher lost mass than the other collisions, since the
GMC process involves a succession of bound giant impacts, while a faster HRC collision has only a single encounter.

As the mass ratio $\gamma$ increases, both bodies become similar in size, so that for even a relatively head-on impact angle, only a minor fraction of the impactor and target physically intersect (Asphaug 2010). This is not the case for small $\gamma$, where for all but the most grazing angles, the target blocks the entire impactor, transitioning to the cratering regime. In the specific cases considered here, assuming spheres passing through spheres, half of the impactor volume intersects the target volume for an impact angle of $37^{\circ}$ with the $0.2 M_{\oplus}$ impactor and $33^{\circ}$ for the $0.5 M_{\oplus}$ one. These are more head-on than the average collision yet still count as "grazing" due to the similarsized geometry. The transition between grazing and nongrazing happens at approximately these angles, with a window of about $10^{\circ}$ on steeper impact angles where GMCs still occur and steeper angles transitioning to more direct (although never perfect) mergers.

The impact angle for which the regime change between GMCs and HRCs occurs depends on the mass ratio and velocity (Kokubo \& Genda 2010); for low-velocity collisions (close to the mutual escape velocity), only the most grazing collisions result in HRCs, as a small energy dissipation is sufficient to bind the bodies. On the other hand, higher-velocity collisions need to dissipate more energy, requiring a steeper impact angle to be collisionally captured. In this work, we focus on collisions around $45^{\circ}$ because they are the most common (Shoemaker 1962) and the transitions are observed to occur around this angle.

Our results are in agreement with the general outcome from Kokubo \& Genda (2010). There is, however, a small discrepancy concerning the dependence on the impact angle with the mass of the bodies. In our results, we observe a slight shift toward lower angles as the mass ratio increase for any velocity. In our results, there are no simulations that are in different regimes in the sets with $m_{\mathrm{imp}}=0.2$ and $0.5 M_{\oplus}$ for the same impact velocity and angle; however, the regime change can be inferred from the departing velocity. We note that overall, the departing velocity given in terms of the escape velocity of the resulting bodies is lower for the higher mass ratio $\gamma=0.56$ than for the lower value $\gamma=0.22$ for the otherwise same initial conditions. The regime change occurs at $v_{\text {dep }} / v_{\text {esc }}=1$, as the difference between GMCs and HRCs is essentially whether the impactor's remnant is bound to the target past the first encounter. So, the transition is pushed by a small amount toward lower angles for a given impact-to-escape velocity ratio as the mass of the impactor increases. This implies that HRCs are slightly less likely for more equal-size bodies, independent of the impact velocity. 


\subsubsection{Material Transfer during Collision}

The HRCs lead to material exchange, with a net result of mass transfer from the impactor to the target, and sometimes vice versa. In one case, we obtain a runner whose mantle is composed of $18.9 \%$ target material. Mass transfer from the projectile to the target can also be more substantial but is diluted by the mass ratio of the collision. We find that up to $9.0 \%$ of the final target's mantle can be composed of impactor material in the $m_{\mathrm{imp}}=0.2 M_{\oplus}$ collisions, and for the more massive targets, $m_{\mathrm{imp}}=0.5 M_{\oplus}$, they can end up with as much as $10.2 \%$ impactor material. So, after one HRC, the remnants have a cosmochemical correlation at the $10 \%$ level that they did not share before the collision. Mass transfer is generally only important for collisions at steep angles, more head-on, as these lead to the greatest interactions between the bodies. Only highvelocity collisions are able to produce HRCs at steep angles, as they otherwise fall into the GMC regime. Low-velocity HRCs are limited to shallow impact angles, where only minimal material interaction occurs, and are therefore unable to provide significant mixing.

We note a smaller effect on the metallic cores. Since cores are deep down in the body, they are more difficult to reach by the shock and require steeper angles for any material interaction. As such, it is nearly impossible to extract target core material, and we have not identified any case where target core material is transferred to the runner. The opposite, however, does occur, where the impactor is so thoroughly shredded by the target that some of its core stays behind. In the simulations with $m_{\mathrm{imp}}=0.2 M_{\oplus}$, the target accreted up to $5.2 \%$ of the impactor's core. For the cases without any transfer, both were for the most grazing collisions we modeled in this study, $\theta_{\text {coll }}=60^{\circ}$. For the simulations with $m_{\text {imp }}=0.5 M_{\oplus}$, only two show some transfer, both with $\theta_{\text {coll }}=45^{\circ}$, with a values of less than $1 \%$. The small mass ratio in the former case makes it easier for the target to extract the impactor's core compared to the latter case. This implies that there is an optimum mass ratio for core transfer onto the target. We estimate that the lowermass impactors are close to the most efficient location; hence, target's core would not be diluted by more than $10 \%$ in the best-case scenario. Higher-velocity impacts allow for more head-on HRCs, which have the potential to allow more impactor cores to be transferred onto the target during such an event.

The most basic net effect is that the mass ratio of the resulting bodies $\gamma_{\text {after }}$ is lower than the pre-collision value $\gamma$. As most of the material transfer involves mantle material, the core mass fraction of the runner is increased. This effect has been invoked to explain Mercury's anomalous high density (Asphaug \& Reufer 2014), where successive collisions would leave a runner highly depleted of its mantle. As mantle stripping of the runner requires a high-mass contrast, such an HRC would have a minor effect on the core mass fraction of the target, primarily by contributing stripped impactor silicate onto the target, both right away (as modeled here) and as a longer sweep-up (Asphaug 2017).

\subsubsection{Mass Loss}

We track only the largest and second remnant of each collision. That is because the collisions involved in this work are still in the low-velocity regime so that no fragmentation occurs, just mass loss in the form of stripping and shedding of unresolved materials. The mass of the tertiary remnants, $m_{\text {lost }}$, that we neglect is always less than $1 \%$ of the total mass involved for the collisions studied here. We note that the GMCs usually eject a higher mass fraction of sparse material than the HRCs. This is because the ejected material carries a part of the angular momentum away, which in the case of GMCs must be released, as the main body cannot sustain the resulting spin (e.g., Asphaug \& Reufer 2013). In HRCs, the runner carries away a significant part of the angular momentum; therefore, there is no need to eject material for this purpose. Since the mass loss is small and the ejection velocity is smaller than the impact velocity, the resulting equivalent of the impact angle $\theta_{\text {dep }}$ must increase to achieve angular momentum conservation.

\subsection{Dynamical Evolution}

\subsubsection{Destination}

For the HRC simulations presented in Table 1, we perform a dynamical evolution of the main remnants. Our interest is in the destination of the runner, i.e., whether a further collision occurs, and if this is the case, the involved bodies (a return collision between the same bodies, or between ones) plus the relevant impact conditions. We are also interested if there is a relationship between the impact parameters of the successive collisions, including their relative orientations. To provide a realistic environment of the late stage of planetary formation, we explicitly include other solar system bodies in the simulation: Mercury, Venus, Mars, Jupiter, and Saturn, with their present-day characteristics. This can be regarded as representative of past planetary characteristics, although we have not yet studied other configurations. For comparison, we also compute the identical dynamical evolution but without these additional bodies present. The resulting occurrences are provided in Table 3.

With the more realistic case of the addition of planets, the fraction of runners that make a second collision with the largest remnant from the initial collision (the original target) is decreased sharply by nearly a factor of two. As seen in the figure, the value is actually quite dependent on the departing velocity; the lowest-velocity runners $\left(m_{\mathrm{imp}}=0.2 M_{\oplus}\right.$, $v_{\text {coll }} / v_{\text {esc }}=1.10, \quad \theta_{\text {coll }}=55^{\circ} \quad$ with $\quad v_{\text {dep }} / v_{\text {esc }}=1.01 \quad$ and $m_{\mathrm{imp}}=0.2 M_{\oplus}, \quad v_{\text {coll }} / v_{\mathrm{esc}}=1.20, \quad \theta_{\text {coll }}=42^{\circ} .5 \quad$ with $\left.v_{\text {dep }} / v_{\text {esc }}=1.00\right)$ have the highest return likelihood, with $61.0 \%$ and $60.7 \%$, respectively. On the other hand, the fastest escaping runners have the lowest return probability; in the case of $\quad m_{\mathrm{imp}}=0.2 M_{\oplus}, \quad v_{\text {coll }} / v_{\mathrm{esc}}=1.20, \quad \theta_{\text {coll }}=52^{\circ} .5 \quad$ with $v_{\text {dep }} / v_{\text {esc }}=1.10$, only $27.6 \%$ collide again with the same body within $20 \mathrm{Myr}$. In this situation, we actually see a greater likelihood of collision with Venus, at $29.6 \%$, indicating that for certain accretion regimes, Earth and Venus could have shared some common aspects of their giant impact evolution. And, quite noticeably, the intersection at $v_{\text {inf }}=0$ is lower than unity, at about 0.7 , so that the argument that runners mostly return to the same body is not correct, even just beyond the transition from GMCs to HRCs.

In Figure 3, we provide the fraction of returns versus the relative velocity at infinity, as determined by the two-body problem with $v_{\text {inf }}^{2}+v_{\text {esc }}^{2}=v_{\text {dep }}^{2}$. The principal dependency seems to be on the remaining velocity, although we do note slight shifts for different impactor masses and orbital configurations. It should be noted that the escape velocities are not identical between the different points, as the value employed 
Table 3

Fractions of Destination of the Runner after 20 Myr of Dynamical Evolution

\begin{tabular}{|c|c|c|c|c|c|c|c|c|c|c|}
\hline$m_{\mathrm{tar}}\left[M_{\oplus}\right]$ & $m_{\mathrm{imp}}\left[M_{\oplus}\right]$ & $\gamma$ & $v_{\text {coll }} / v_{\mathrm{esc}}$ & $\theta_{\text {coll }}[\mathrm{deg}]$ & $f_{\text {Tar }}$ & $f_{\text {Mercury }}$ & $f_{\text {Venus }}$ & $f_{\text {Mars }}$ & $f_{\text {Other }}$ & $f_{\mathrm{Rem}}$ \\
\hline \multirow[t]{11}{*}{0.9} & 0.2 & 0.22 & 1.10 & 55.0 & 0.558 & 0.012 & 0.188 & 0.016 & 0.067 & 0.159 \\
\hline & & & & & 0.953 & $\ldots$ & $\ldots$ & .. & $\ldots$ & 0.047 \\
\hline & & & 1.10 & 60.0 & 0.442 & 0.009 & 0.238 & 0.015 & 0.092 & 0.204 \\
\hline & & & 1.15 & 52.5 & 0.360 & 0.018 & 0.306 & 0.015 & 0.087 & 0.214 \\
\hline & & & & & 0.936 & $\ldots$ & $\ldots$ & $\ldots$ & $\ldots$ & 0.064 \\
\hline & & & 1.15 & 60.0 & 0.325 & 0.025 & 0.259 & 0.037 & 0.120 & 0.234 \\
\hline & & & & & 0.968 & $\ldots$ & $\ldots$ & $\ldots$ & $\ldots$ & 0.032 \\
\hline & & & 1.20 & 45.0 & 0.405 & 0.014 & 0.285 & 0.016 & 0.078 & 0.202 \\
\hline & & & & & 0.937 & $\ldots$ & $\ldots$ & $\ldots$ & $\ldots$ & 0.063 \\
\hline & & & 1.20 & 52.5 & 0.264 & 0.021 & 0.266 & 0.042 & 0.142 & 0.265 \\
\hline & & & & & 0.895 & $\cdots$ & $\ldots$ & $\ldots$ & $\ldots$ & 0.105 \\
\hline \multirow{7}{*}{0.9} & & & & & 0.817 & $\ldots$ & $\ldots$ & $\ldots$ & $\ldots$ & 0.183 \\
\hline & & & 1.20 & 45.0 & 0.425 & 0.019 & 0.208 & 0.022 & 0.245 & 0.081 \\
\hline & & & & & 0.852 & $\ldots$ & $\ldots$ & $\ldots$ & $\ldots$ & 0.148 \\
\hline & & & 1.20 & 52.5 & 0.301 & 0.023 & 0.247 & 0.029 & 0.290 & 0.110 \\
\hline & & & & & 0.776 & $\ldots$ & $\ldots$ & $\ldots$ & $\ldots$ & 0.224 \\
\hline & & & 1.20 & 60.0 & 0.244 & 0.015 & 0.265 & 0.026 & 0.341 & 0.109 \\
\hline & & & & & 0.724 & $\ldots$ & $\ldots$ & $\ldots$ & $\ldots$ & 0.276 \\
\hline
\end{tabular}

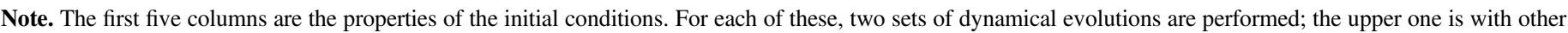

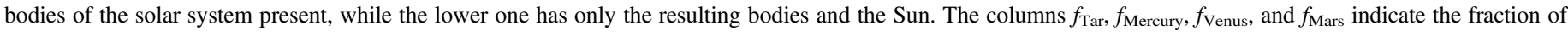

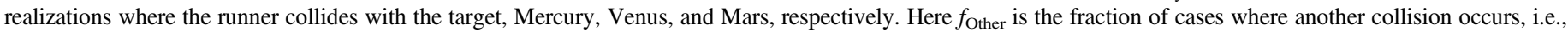
either between the largest remnant and another planet or between two other planets, and $f_{\text {Rem }}$ is the fraction where no collision occurs within 20 Myr.

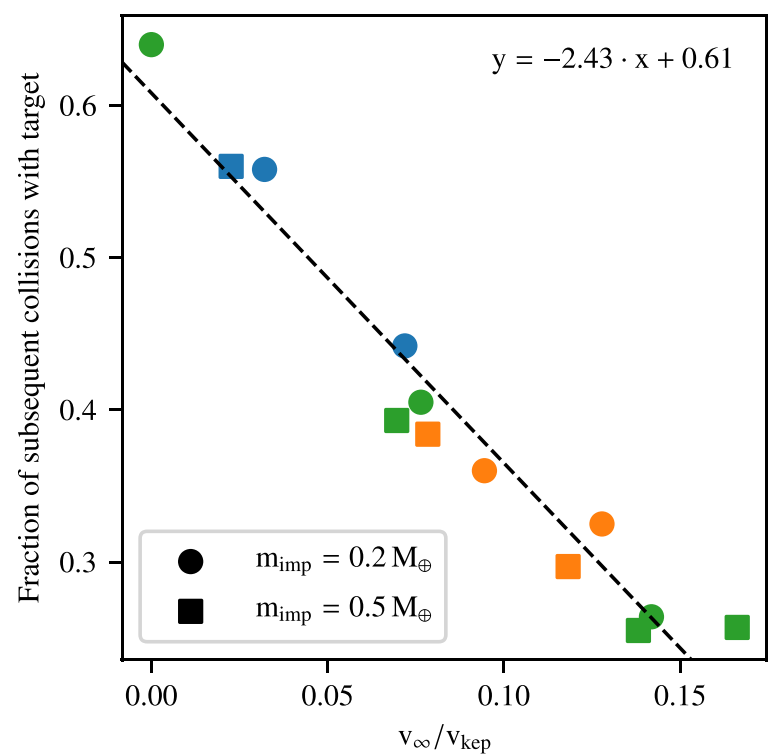

Figure 3. Fraction of runners that make a subsequent collision with the target within 20 million yr for each set of dynamical evolution calculations as a function of the relative velocity at infinity. The shape of each symbol denotes the impactor's mass, with circles for $m_{\mathrm{imp}}=0.2 M_{\oplus}$ and squares for $m_{\text {imp }}=0.5 M_{\oplus}$, and the color denotes the velocity of the initial collision, with blue for $v_{\text {coll }} / v_{\text {esc }}=1.10$, orange for $v_{\text {coll }} / v_{\mathrm{esc}}=1.15$, and green for $v_{\text {coll }} / v_{\text {esc }}=1.20$. The dashed black line provides the least-squares fit of all points, whose parameters are provided in the top right corner. Of the runners that barely escape the collision, only two-thirds return to the target. For characteristic encounter velocities, between $1 / 3$ and $2 / 3$ of them return, decreasing with $v_{\text {coll }} / v_{\text {esc }}$. here is that of the remaining bodies after the collision, which varies due to differences in mass transfer that occur during the encounter. So $v_{\mathrm{esc}}$ is always normalized, meaning also that these results can with some degree of confidence be applied to larger and smaller mass regimes, depending on the sensitivity of the problem to the equation of state.

Evidently, just beyond the transition from GMCs to HRCs, there is a fraction (around 1/3) of lost runners that become detached from the target. This detachment is more efficient in the presence of other planets. To demonstrate this, we performed the same suite of dynamical evolution studies but using the longest-period GMC collision, $m_{\mathrm{imp}}=0.2 M_{\oplus}$, $v_{\text {coll }} / v_{\text {esc }}=1.10, \theta_{\text {coll }}=52^{\circ} .5$ (see Table 2 ). In roughly $75 \%$ of the cases, the secondary body returns after the time obtained from the two-body problem. The remainder take longer to return, with up to $10 \mathrm{yr}$, that is, more than two orders of magnitude more than the expected value. This means that with some orbital configuration, the trajectory of the runner is affected by the presence of other planets, and the GMC is close to becoming an HRC.

In the case of an impact on the Earth, the transition region between GMCs and HRCs is narrow. There is only a small window where the impactor is captured but on a sufficiently wide orbit to be affected by the other bodies. From the two simulations performed for $m_{\mathrm{imp}}=0.2 M_{\oplus}$ and $v_{\text {coll }} / v_{\mathrm{esc}}=1.10$ that are 2.5 apart, one is a GMC with the secondary body always returning, and the other is an HRC with $v_{\text {dep }} / v_{\text {esc }}=1.01$; so, in the case of an Earth-like orbit, this window is abrupt, on the order of $1^{\circ}$. 
The other possibilities behave in the same inverse trend. The decrease of the accretion rate of the runner with increasing departing velocity is reminiscent of the growth in the oligarchic regime, where the rate decreases as the eccentricities and inclinations of the smaller bodies increase (Inaba et al. 2001; Chambers 2006). The departing velocity of the runner is a proxy for the eccentricity of its orbit, so the most rapidly departing ones will spend the lower part of their time in a region where the runner is, while being more likely to reach the other bodies in the system.

We also observe collisions between other bodies than the runner or the target. The fraction of systems that undergo such events is indicated in the column $f_{\text {Other }}$ of Table 3 . It could be that the runner collides later on, but as we do not yet have a consistent collision model in the $N$-body, the state of the system is undetermined. To avoid artifacts in this situation, we halt those simulations at the first collision.

We do not observe any collisions with the central star. Ejections occur in 1\%-2\% of the cases. Most of the time, Mars is the ejected planet, while the runner suffers from the same fate in $0.5 \%$ of the cases, on average. Ejection from the solar system following one close encounter with a terrestrial planet is difficult, because the velocity kick provided by such an encounter (which relates to the surface velocity of the planet) is much lower than the escape velocity from the system (e.g., Thommes et al. 2003; Ida \& Lin 2004). A possible path toward ejecting the runner is to have it encounter Jupiter, which can provide a sufficient kick to eject the body from the system (Jackson et al. 2018). However, the velocity resulting from the collision and the further encounters with other bodies are not sufficient to make the runner cross the orbit of Jupiter. Collision with the central star is also unlikely for a similar reason.

The results for the evolution with no other bodies than the collision remnants and the central star are somewhat different. Overall, the return rate is quite high, more than $90 \%$, except in specific cases. Thus, the assumption that the runner returns to the same body is valid only without other objects present to perturb its orbit. Also, the return rate seems to relate more to the initial collision velocity than the departing velocity.

\subsubsection{Relationship with Initial Conditions}

We investigate if specific outcomes are linked to specific initial conditions. For the case with $m_{\mathrm{imp}}=0.2 M_{\oplus}$, $v_{\text {coll }} / v_{\text {esc }}=1.10$, and $\theta_{\text {coll }}=60^{\circ}$, we plot the type of event obtained with respect to the semimajor and eccentricity map in Figure 4. There is no obvious correlation between specific preimpact orbits and the type of event obtained after the collision. The same applies to the other sets of dynamical evolution, as in the case where no other bodies are present.

We do not find any restrictions on the pre-impact orbits of runners that further collide with other bodies. It is then possible for a body coming from the outer part of the system to produce an HRC and end up colliding further in the inner part of the system, as well as a body to be sent back to its originating part. The same also applies for bodies coming from the inner part of the system. The HRCs are then able to redistribute material in the system in the same way close encounters are capable.

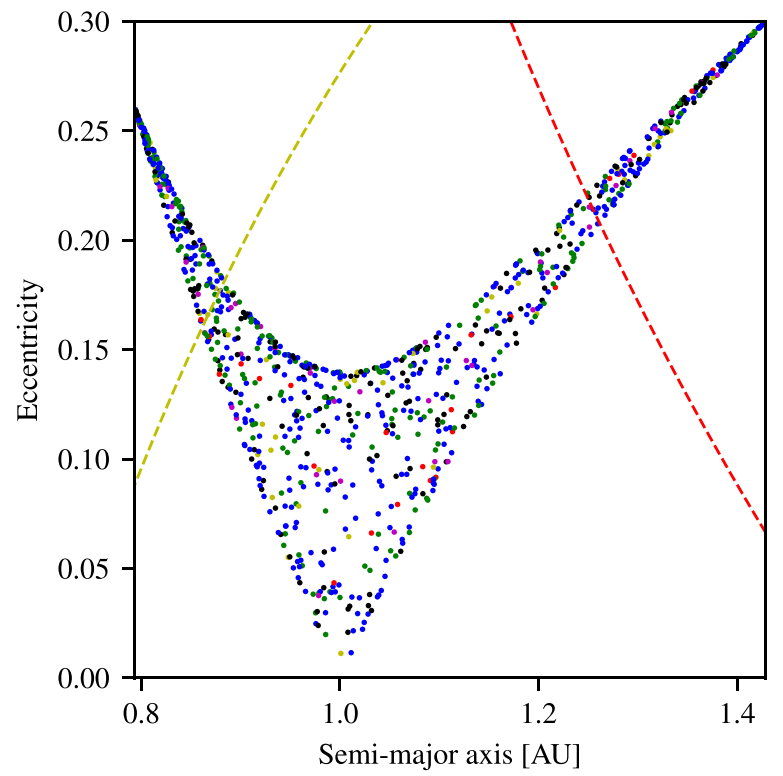

Figure 4. Map of outcome with respect to orientation of the initial collision for the case with $m_{\mathrm{imp}}=0.2 M_{\oplus}, v_{\mathrm{coll}} / v_{\mathrm{esc}}=1.10$, and $\theta_{\text {coll }}=60^{\circ}$. The colors are black for no further collision, blue for a collision between the runner and the target, green for a collision between the runner and Venus, red for a collision between the runner and another body, yellow for a collision between the target and another body than the runner, and magenta for another event. The yellow dashed line denotes Venus-crossing pre-impact orbits, and the red dashed line shows Mars-crossing pre-impact orbits.

\subsubsection{Time until Subsequent Collision}

We show the cumulative distribution of the time span between the two collisions in Figure 5 for three series of dynamical evolution. In each series, the blue line shows the outcome when the simulation is restricted to the remnant bodies from the collision and the central star, while the orange curves denote the general case, with other solar system-like planets. All curves are normalized by the initial number of dynamical evolution simulations, so the end value of each curve represent the overall likelihood of the case that is provided in Table 3.

We provide in Figure 6 the median return time versus the departing velocity. As for the return occurrence rate, we find that the timing is also dependent on the departing velocity; runners that are the slowest to leave return the fastest. Some runners return as early as a few tens of years, but these are particular cases. Most of them return in a time frame of hundreds of thousands to a million years. The correlation between the departing velocity and timing is further consistent with the previous discussion; as the departing velocity increases, so does the runner orbit's eccentricity, and with a higher eccentricity, the collision probability decreases. Hence, it takes more time.

The presence of the other bodies barely affects the return timing; their major effect remains the reduction of the overall return rate. Further, we note that they affect the later returns; indeed, the cumulative distributions for the return time match pretty well (except in one case) until about $10^{5} \mathrm{yr}$. This figure does not depend on specific collision parameters or outcomes. The perturbations by the other bodies made on the runner will shift its trajectory over time. The early collisions happen with orbits that are barely disturbed by the other bodies; hence, similar collisions are observed in either case.

Collisions with the other bodies do not happen as early as with the target. It takes a similar amount of time for the 

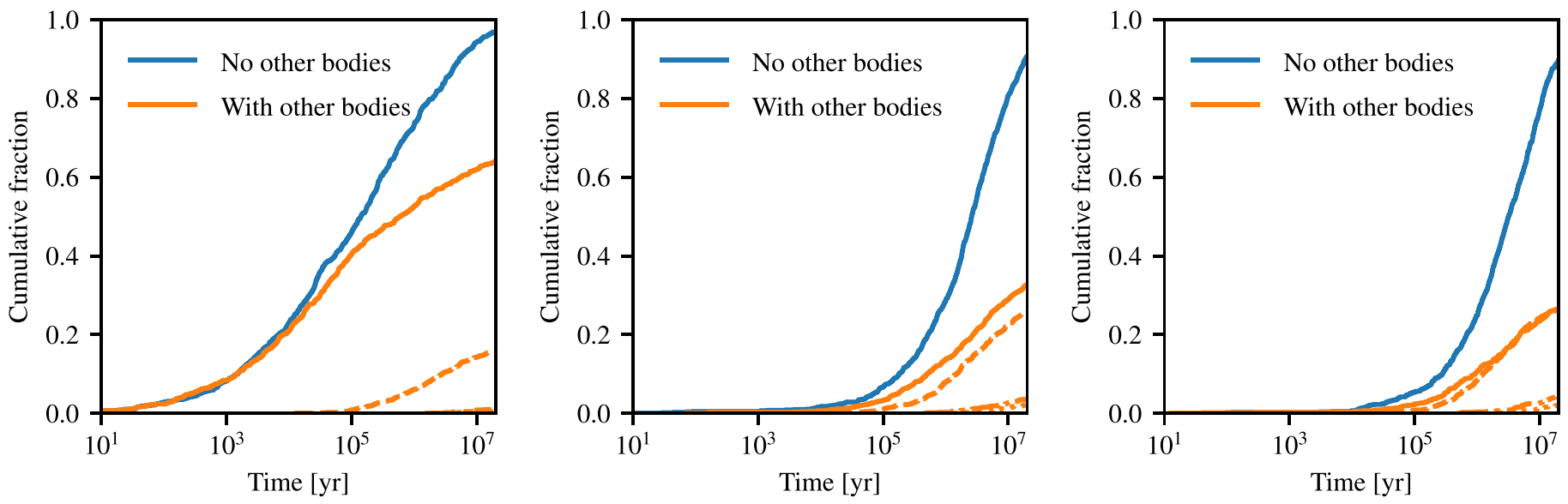

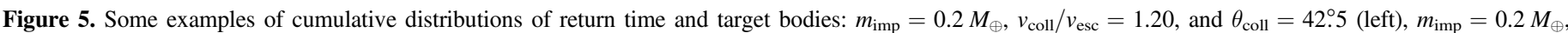

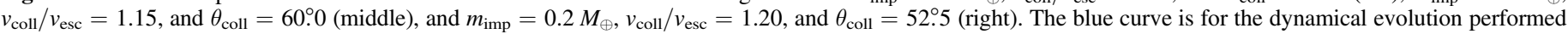

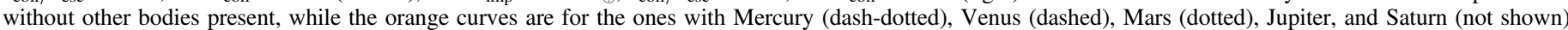

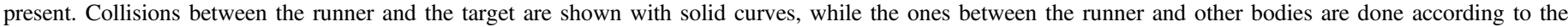
previous description.

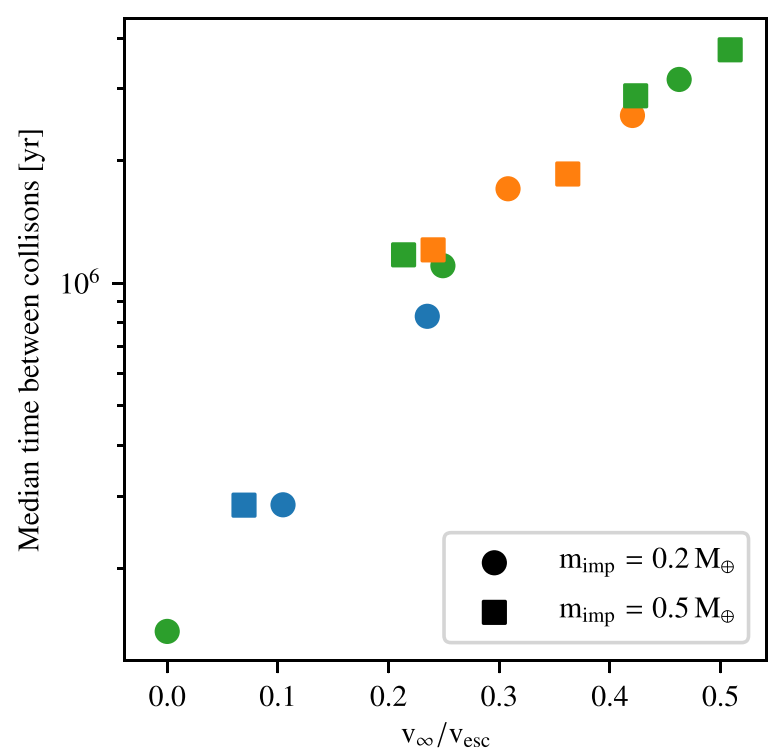

Figure 6. Median time between successive collisions for each set of dynamical evolution calculations as a function of the relative velocity at infinity for all dynamical evolution models without other bodies present. Symbols are the same as in Figure 3: the shape denotes the impactor's mass, with circles for $m_{\text {imp }}=0.2 M_{\oplus}$ and squares for $m_{\text {imp }}=0.5 M_{\oplus}$, and the color denotes the velocity of the initial collision, with blue for $v_{\text {coll }} / v_{\mathrm{esc}}=1.10$, orange for $v_{\mathrm{coll}} / v_{\mathrm{esc}}=1.15$, and green for $v_{\text {coll }} / v_{\mathrm{esc}}=1.20$.

perturbations to reduce the rate of collisions with the target, around $10^{5} \mathrm{yr}$, before the first collisions with Venus occur.

\subsection{Return Collision Parameters}

We further analyze the return collision properties, which are generally the velocity and angle. In our case, we will extend the analysis to the relationship between the successive collisions, as well as the orbital configuration. This ultimately enables us to determine their regime, i.e., whether these are HRCs again or mergers.

For reference, we modeled-by mistake - a series of dynamical evolutions where all of the initial collisions were assumed to happen vertically, i.e., $\theta \approx 0$. In the case of subsequent events and timing, it behaves as for coplanar impacts; however, it will be useful further on, as it provides insight on the effect of geometry on the results.

\subsubsection{Velocity}

The velocity of the collision is a key parameter determining the outcome, e.g., whether it is an HRC, a disruption, a GMC, or a merger. Thus, the velocity of the returning collision is of key interest in determining the final evolution of a giant impact chain, whether the chain continues (another HRC) or ends (a merger). In this section, we correlate the incoming velocity for the first collision with the return velocity of the second collision. For example, for immediate returns, the return velocity is expected to be close to the departing velocity. But when the return collision comes later, mutual interactions might affect the trajectory, leading to different impact velocities. Therefore, the results are shown as $2 \mathrm{D}$ histograms of impact velocity versus delay until the subsequent collision occurs.

We show such results for three sets of dynamical evolutions in Figure 7. The impact and departure velocities of the initial collision are marked with red and black dashed lines, respectively. We do observe a few features: the return velocity is more related to the departing velocity than the initial velocity, and as the time between the collisions increases, so does the spread in impact velocities. The cases shown here represent the variety of outcomes obtained in this study. The first case is where the departing velocity is close to the mutual escape velocity; i.e., the collision is close to the transition between HRCs and GMCs. Only the case with the other solar system bodies is shown, as without them, all return events occur at the same velocity. The second and third cases show a departing velocity much higher than the mutual escape velocity, where the spread of the return collision velocity can be obtained in both directions. With other bodies present, we note that there are a few events with $v_{\text {coll }} / v_{\mathrm{esc}}>1.25$, which is not the case otherwise. Nevertheless, as the departing velocity is lower than the initial collision velocity, when see that there is only a small fraction of the return collision occurring at a higher velocity than the initial one. The velocity decrease is less pronounced as the impact angle of the initial collision increases; the collision dissipates less energy for grazing events, so the departing velocity is closer to the initial impact 

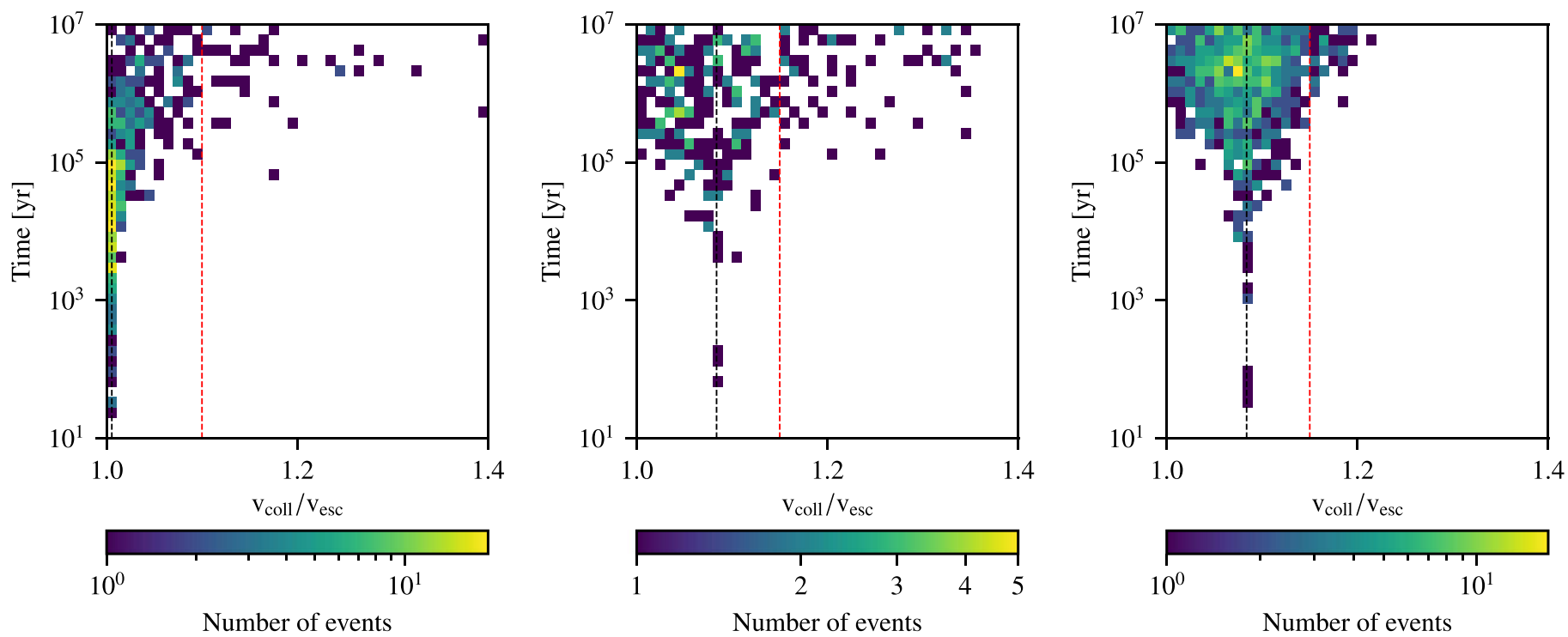

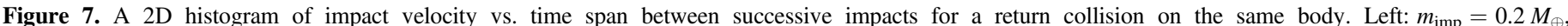

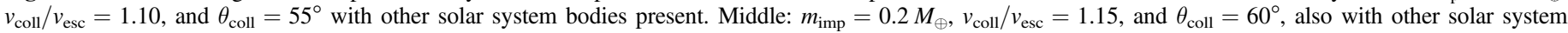

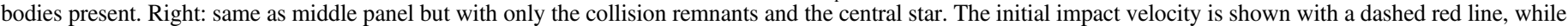
the impact departure velocity is shown with a dashed black line.

velocity. The same applies for the return velocity, which relates to the departing velocity.

The relationship between the departing and return velocity is quite straightforward. If energy was conserved in the point of view of two bodies (target and runner), then the return collision velocity would happen with the same velocity. With other bodies, close encounters lead to modifications of the orbits, and the return velocity can vary. The presence of other bodies provides more means of exchanges; therefore, the impact velocity spread is higher in this situation.

Second, the higher spread over time is linked to the same effect. As time goes on, perturbations by the other bodies increase. The early returns happen at a velocity very close to the departing velocity. After again roughly the same time as given in the previous section, $10^{5} \mathrm{yr}$, the impact velocities start to diverge noticeably. In the case with other bodies present, the median of the distribution remains at the departing velocity.

To properly resolve the return collision, correct determination of the departing velocity from the initial collision is required, as this is what determines the properties of the second event. Assuming that the runner leaves the target with essentially the same velocity as the one it came with neglects various physical processes, among them energy dissipation through shocks.

Since in the cases with a relatively large departing velocity, many collisions between the runner and Venus are obtained, we perform the same analysis for that series of events. The result is shown in Figure 8. It should be noted that the axes are not showing the same values as in Figure 7; the time axis has been shrunk to show only the later times, as no early collisions occur, and the velocity axis has been expanded, as there are only a handful of events that happen outside of the range shown here. We observe a similar trend that the return collision velocity increases over time. However, the base velocity does not seem to be related to the departing velocity of the prior collision; rather, it seems to be somewhat lower at around $v_{\text {coll }} / v_{\text {esc }} \approx 1.05$. In Figure 8, the velocity has been corrected to

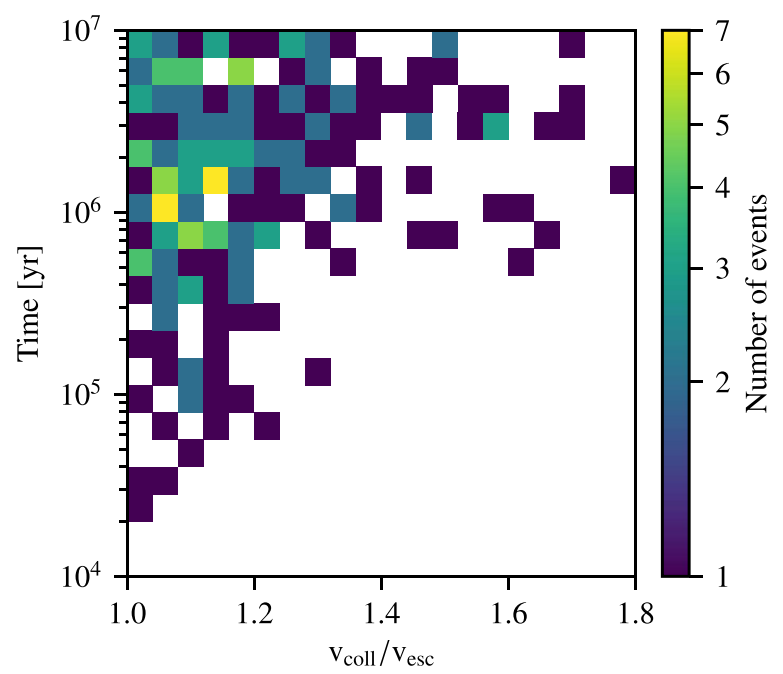

Figure 8. A $2 \mathrm{D}$ histogram of impact velocity vs. time span between a first collision hitting the target and a second collision hitting Venus. Here $m_{\mathrm{imp}}=0.2 M_{\oplus}, v_{\mathrm{coll}} / v_{\mathrm{esc}}=1.15$, and $\theta_{\text {coll }}=60^{\circ}$.

account for the different mass and radius of the Venus body compared to the end state of the target.

If we follow the methodology from Jackson et al. (2018) again, the minimum relative velocity at Venus of an impactor that has an Earth-crossing orbit (assuming both Venus and Earth are on circular orbits) results in an impact velocity of $v_{\text {coll }} / v_{\text {esc }} \simeq 1.045$. This value is very close to the one reported above, so the results are consistent. The same orbit requires a relative velocity at Earth's orbit of $v_{\text {rel }} / v_{\text {esc }} \simeq 0.27$, or $v_{\text {dep }} / v_{\text {esc }} \simeq 1.04$. This implies that a runner departing with a lower velocity cannot have directly Venus-crossing orbits and requires interactions with other bodies to become so. As the departing velocity increases, so does the fraction of orbits that can be Venus-crossing (Figure 1).

We also obtained collision with the Mercury and Mars analogs; however, the number of those events is too low to obtain meaningful statistics. 

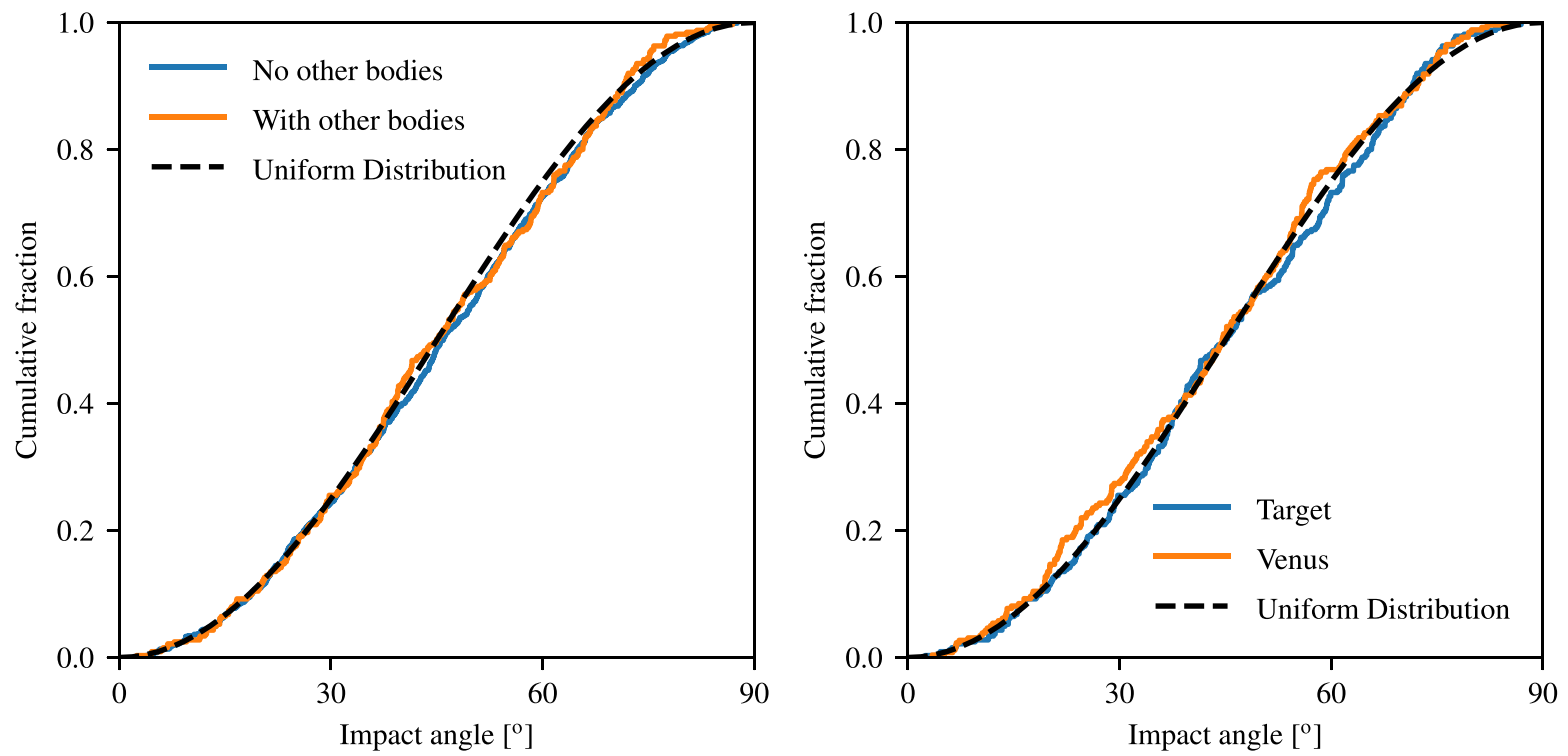

Figure 9. Cumulative distribution of impact angles for collision between the runner and target (left) and the runner and Venus (right). Results are from the dynamical evolution following the collision with $m_{\mathrm{imp}}=0.2 M_{\oplus}, v_{\text {coll }} / v_{\mathrm{esc}}=1.15$, and $\theta_{\text {coll }}=60^{\circ}$. The black dashed line is the expected distribution if the offset of the runner follows a uniform distribution, computed following Shoemaker (1962).

\subsubsection{Impact Angle}

The basic collision geometry is set by the impact angle. Its cumulative distribution for the return collisions with the target in one dynamical evolution series, in the cases both with and without additional bodies, is shown in Figure 9. In addition, we provide a theoretical distribution of the impact angles if the impactors were uniformly approaching the target following Shoemaker (1962). In the latter case, the mean, median, and mode of the distribution are all located at $45^{\circ}$.

For all of the dynamical evolution models performed, with additional bodies present or not, we obtain very similar results: the distribution of the impact angles of the return collision is compatible with the uniform distribution. To quantify this, we run a one-sample Kolmogorov-Smirnov (K-S) test to asses the compatibility of the results with the reference distribution. All but one obtained $p$-value are greater than $3 \%$, so we have no strong argument to reject that the obtained distribution derives from a uniform distribution. The exception is the case with $m_{\mathrm{imp}}=0.2 M_{\oplus}, \quad v_{\text {coll }} / v_{\mathrm{esc}}=1.20$, and $\theta_{\text {coll }}=52^{\circ} .5$, which shows an excess of collisions with $\theta \approx 30^{\circ}$.

The compatibility with a uniform distribution implies that the end geometry of the prior collision has no influence whatsoever on the subsequent event, and in this direction, the fact that the same bodies had already collided has no importance. Since the target-runner pair is not an isolated system, there is no reason to expect that angular momentum would be conserved, which would be needed in order to set a constraint on the impact angle of the return collision. Since HRCs are happening for grazing angles, the return collision has, on average, a steeper impact angle.

The same compatibility with uniform distribution is also obtained for collisions with other bodies. This is not much of a surprise because, if the distribution of impact angles for a return collision with the same body is compatible with a uniform distribution, it would be hard to understand why this would not be the case for other bodies.

Lastly, there is a mismatch for the set of dynamical evolutions where all of the collisions were modeled as occurring in the orbital plane. If all collisions happen in the same plane, then a different distribution of impact angles, shifted toward steeper values, is expected (Emsenhuber 2017; Leleu et al. 2018). In the latter case, more head-on collisions are favored, and the median is located at $30^{\circ}$, compared to $45^{\circ}$ in the general case following Shoemaker (1962). In our case, we observe a slight increase of more head-on events, though the distribution of impact angles is closer to the general distribution than the one for impacts in one plane. As we shall discuss further on, the other properties of this dynamical evolution set tend to the expected distributions for impacts uniformly oriented in space. As to why, we can say that our SPH simulations are not exactly aligned in the direction orthogonal to the impact plane; the relative motion of the two remnants is inclined by nearly $1^{\circ}$. This small deviation is sufficient to induce vertical motion of the bodies that results in a uniform distribution of impact angles in a volume rather than in a plane. Only a small vertical deviation in the vertical direction is needed to obtain the general distribution, slightly greater than $r_{\perp}$. We calculated that the required inclination to fulfill this condition is on the order of $10^{-3}$. Since the return velocity is somewhat smaller than in the initial collision, $r_{\perp}$ is increased; however, the value remains small enough to have a uniform-in-space-like distribution.

\subsubsection{Alignment between Successive Collisions}

In the cases in which the runner returns to the target, there is a series of two collisions between the same bodies. It is then possible to search for relationships between these events, such as the spatial alignment of the impact planes. Since HRCs are grazing events, a part of the relative angular momentum is transferred during the collision from the orbital motion into spin. If we assume that the spin axis is not modified between the collisions, then the alignment between the relative angular momentum vectors is a proxy for the alignment of the returning runner and the spin of the bodies. The results for one series of dynamical evolution are provided in Figure 10; the other series behaves similarly. 


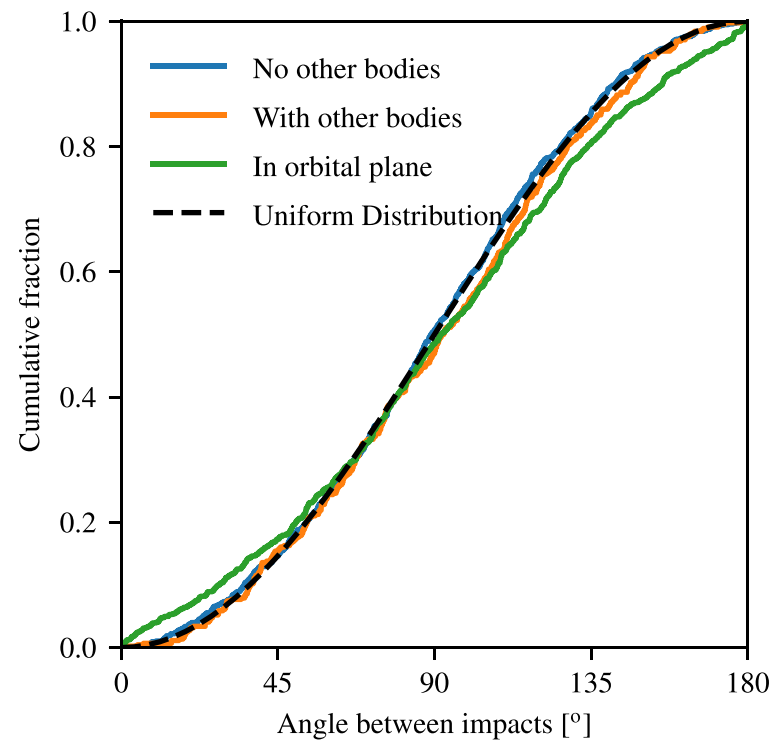

Figure 10. Cumulative distribution of angles for collisions between the runner and target. Results are from the dynamical evolution following the collision with $m_{\text {imp }}=0.2 M_{\oplus}, v_{\text {coll }} / v_{\text {esc }}=1.10$, and $\theta_{\text {coll }}=60^{\circ}$. The black dashed line is the expected distribution if the offset of the runner follows a uniform distribution.

As for the impact angle, we find that the angle between the specific angular momentum vectors is compatible with a uniform distribution, except for the cases where the initial collision is assumed to happen in the orbital plane. The lowest $p$-value obtained (with the exception of the in-orbital-plane configuration) is $2 \%$, with an even distribution of values up to unity.

The case where all collisions are assumed to be in the orbital plane is special, as we observe an excess of collisions occurring with a relative angle close to either $0^{\circ}$ or $180^{\circ}$, i.e., also in the same plane, with prograde and retrograde alignment, respectively. This is again consistent with the previous result about impact angle, as the collisions with relative angle happen close to the orbital plane. This seems to be restricted to the specific case of collisions in the orbital plane, however. In our other set, where there are only almost-vertical impacts, we do not find such a correlation in the angles between successive collisions; the distribution is similar to the ones from the general case and compatible with a uniform distribution. Coplanar collisions are special, in the sense that angular momentum is almost perpendicular to the orbital plane; therefore, vertical motion is limited. It has a negligible effect on the overall distribution, as the probability of obtaining such a case-or a close-by one - is infinitesimal: the probability of a certain inclination is $d P \propto \cos i d i$, assuming a uniform distribution of the specific angular momentum, and $\cos (-\pi / 2)=\cos (\pi / 2)=0$. Therefore, we do not find any evidence for a correlation between the alignment of successive collisions between the same bodies, except in specific circumstances that require some external mechanism-e.g., inclination damping by a gas disk- to be attained.

If the orientation of the previous collision relates to the spin axis of the bodies prior the return collision, then no specific configuration is favored. Most of the event should occur with the impactor colliding perpendicular to the spin motion of the target. Whether the impact occurs at the pole or on the equator is unconstrained as well. Note that we usually refer to the spin motion of the target only, though the impactor may be rotating as well, since the target with its larger mass carries an important fraction of the spin angular momentum. Prograde and retrograde collisions have equal likelihood, but it is unlikely that these are well aligned.

The nonalignment makes HRRs quite different from GMCs. In GMCs, the runner returns after a short period of time with the same alignment and angle as the end state of the initial collision. Neither holds in HRRs, so that the overall accretion process behaves differently. Both HRCs and GMCs carry significant angular momentum, usually higher than the spin angular momentum a body can sustain (Chandrasekhar 1969). In the case of GMCs, a part of the material must be ejected, carrying away angular momentum (e.g., Asphaug \& Reufer 2013). For HRRs, there are other ways to limit the final spin angular momentum. First, there is the modification of the impact angle; we saw in the previous section that the impact angle of the return collision is unconstrained by the end state on the initial collision. Since the equivalent of the impact angle is quite large and that of the return collision is, on average, $45^{\circ}$, this leads to a reduction of the angular momentum carried by the orbital configuration. Second, if the return collision occurs perpendicular to the spin axis of the body, then angular momentum is added in quadrature, which leaves a final value lower than if the return collision happens in the prograde direction, which is the case of a GMC. The HRRs can then be more efficient to accrete the returning runner, provided the return collision is a merger, either simple or a GMC.

\subsubsection{Orientation}

To determine the orientation of the collision, we check two vectors: specific angular momentum and relative velocity at infinity (or the eccentricity vector, in the unlikely case of a closed orbit). They are orthogonal by construction, though they do not provide the same information. For instance, an impact occurring in the orbital plane is rare, as we saw in the previous sections. However, a small vertical offset when compared to orbital distance is able to produce highly oblique impacts (see related discussion in Section 2.2). The relative velocity vector at infinity, on the other hand, provides a greater insight on the mutual inclinations, as a nonhorizontal one requires some inclination (Jackson et al. 2018).

The results are shown in Figure 11 for the dynamical evolution following the collision with $m_{\mathrm{imp}}=0.2 \mathrm{M}_{\oplus}$, $v_{\text {coll }} / v_{\text {esc }}=1.10$, and $\theta_{\text {coll }}=60^{\circ}$. While not displayed here, other cases have been performed and show similar results. For the initial conditions that were taken to follow a uniform distribution in space, we do not have sufficient evidence against the results being compatible with the same. We do obtain a $p$ value of $3 \times 10^{-3}$ for the inclination of the relative velocity vector at infinity in one set, but otherwise, the values are much higher. This is not the case when the initial collision is assumed to occur in the orbital plane. The results are not compatible with a uniform, with $p$-values between $10^{-6}$ and $10^{-3}$ for both quantities. Looking in more detail, we note that there is a contribution of roughly $10 \%$ of collisions that happen very close to the orbital plane, as the inclination of the relative velocity vector at infinity is almost zero, and the inclination of the collision plane is low. If we plot a 2D histogram of the angle between the collision plane versus time span between collisions, as in Figure 12, it can be seen that this contribution is from early returns. These events happen on such short 

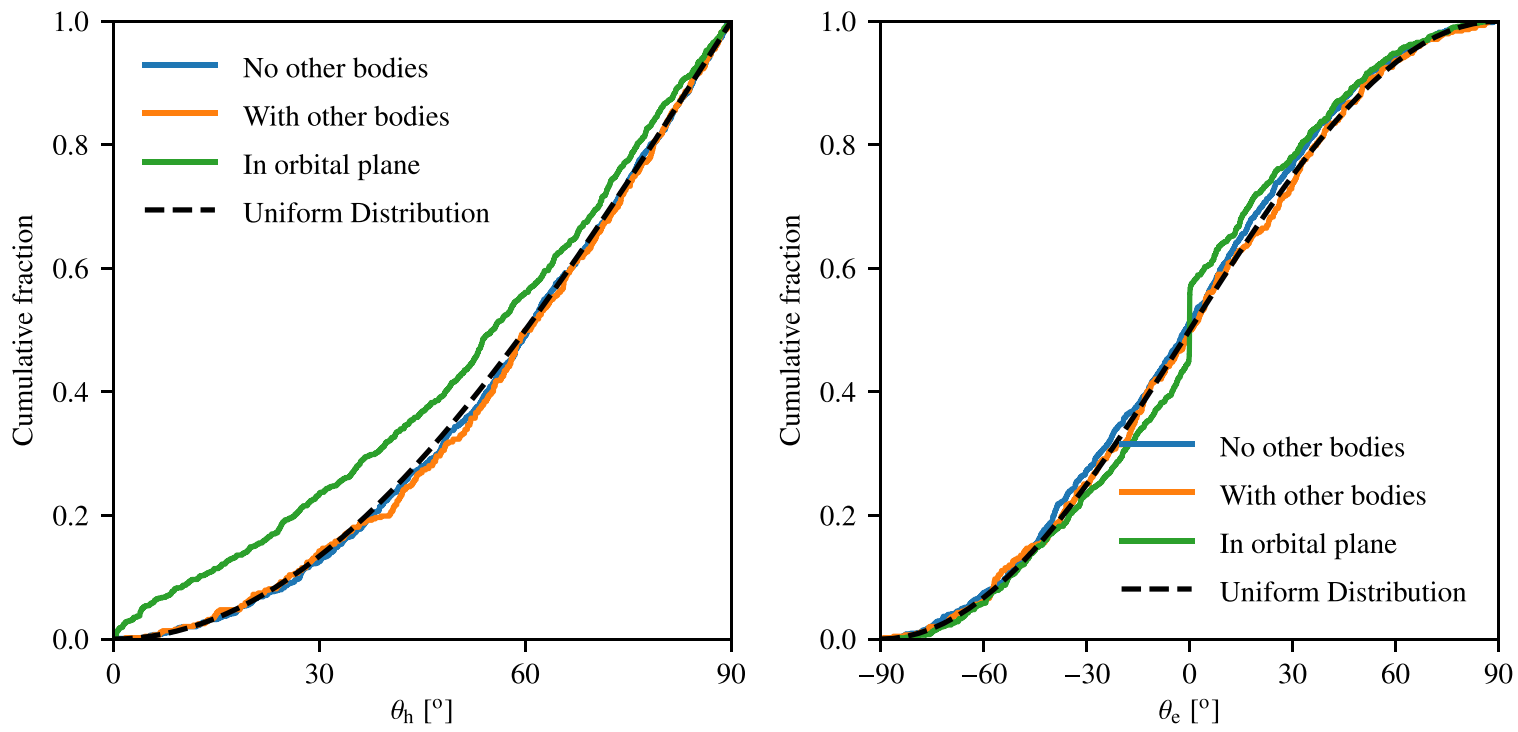

Figure 11. Cumulative distribution of angles for the inclination of the impact plane (left) and the relative velocity vector at infinity (right). Results are from the dynamical evolution following the collision with $m_{\mathrm{imp}}=0.2 M_{\oplus}, v_{\mathrm{coll}} / v_{\mathrm{esc}}=1.10$, and $\theta_{\text {coll }}=60^{\circ}$. The black dashed line is the expected distribution if the offset of the runner follows a uniform distribution.

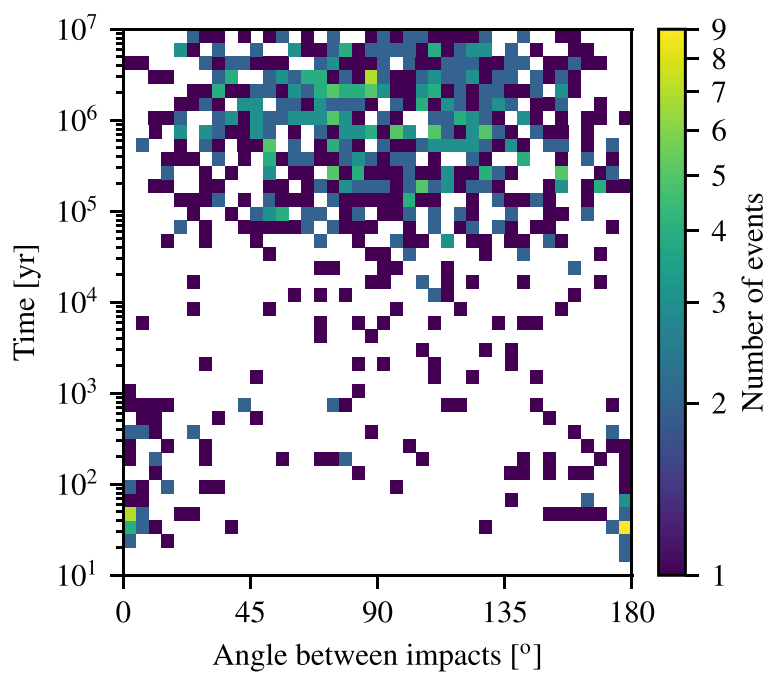

Figure 12. A $2 \mathrm{D}$ histogram of the angle between successive collision and time for the case with $m_{\text {imp }}=0.2 M_{\oplus}, v_{\text {coll }} / v_{\mathrm{esc}}=1.10$, and $\theta_{\text {coll }}=60^{\circ}$ and all initial collisions assumed to occur in the orbital plane. At early times, the orientation of the return collision is correlated to the first collision. Later, the return collision is what one expects from a random distribution of impacts.

timescales, less than $1000 \mathrm{yr}$, that they could not have sufficient close encounters to modify their orbit so that the mutual inclination required for collisions outside of the impact plane is obtained. Also, these early returns are quite evenly distributed between angles close to $0^{\circ}$ (prograde) and $180^{\circ}$ (retrograde). On the other hand, later returns more closely follow the expected values for a uniform distribution, so the noncompatibility is linked to a small set of early return collisions.

These results permit us to determine the relevance of our initial conditions, which we selected so that the relative velocity vector at infinity is uniformly distributed in space. The same distribution is the natural outcome of dynamical evolution. Even when all of the initial collisions happen in the orbital plane, the majority of return collisions follow the same uniform distribution, with the exception of about $10 \%$ of events, due to the specific geometry. We also observe a uniform distribution in our special set that assumes that all initial collisions happen in a near-vertical direction. Therefore, the initial conditions for the dynamical evolution are representative of the expected distribution of impact orientations. Furthermore, we verified that there is no temporal evolution of the distribution. Early collisions are also compatible with a uniform distribution.

\subsection{Effect of Initial Eccentricity}

The initial conditions for the dynamical evolution assume that the target is on a circular orbit prior to the giant impact, and the orbit of the impactor is computed to obtain the desired collision orientation. Since the target is also deflected during a collision, its orbit at the beginning of the dynamical evolution is elliptical. The same applies to the center of mass of the target-impactor system. The trajectory of the center of mass depends principally on the impactor's mass and the relative velocity and only weakly on the impact angle. The cases with the larger impactor, $m_{\text {imp }}=0.5 M_{\oplus}$, therefore have an overall larger eccentricity of remnants than for the smaller one, $m_{\text {imp }}=0.2 M_{\oplus}$.

We check the orbits of the remnants immediately after the collision to see if they are initially crossing with other bodies. Our choice of pre-impact orientation results in similar eccentricities for both remnants; the figures we report here are thus valid for both remnants. In the cases with the smaller impactor, $m_{\mathrm{imp}}=0.2 M_{\oplus}$, the orbits are not initially crossing Venus for the slow-departing runners, while some are for the faster ones, up to $18 \%$. We therefore always obtain more collisions with Venus than there are crossing orbits initially. Mutual excitation is therefore needed to obtain a good part of the collisions with Venus. For the larger impactor, $m_{\text {imp }}=0.2 M_{\oplus}$, the figures of the crossing orbit fractions are similar to those for the smaller impactor at the same velocity and angle, albeit slightly lower. The increased rate is then not due to more eccentric orbits.

To verify the effect of the choice of the initial orbital setup, we perform a second dynamical evolution, where it is assumed that the center of mass is on a circular orbit, for the collision 
Table 4

Type of Return Collisions for Subsequent Collision Obtained in the Dynamical Evolution with the Other Solar System Bodies Present

\begin{tabular}{|c|c|c|c|c|c|c|c|c|c|c|c|c|}
\hline$m_{\mathrm{tar}}\left[M_{\oplus}\right]$ & $m_{\mathrm{imp}}\left[M_{\oplus}\right]$ & $\gamma$ & $\frac{v_{\text {coll }}}{v_{\text {esc }}}$ & $\theta_{\text {coll }}[\mathrm{deg}]$ & $\overline{f_{\text {HRC }}^{\text {ini }}}$ & $f_{\text {Merge }}^{\text {SL12 }}$ & $f_{\mathrm{GMC}}^{\mathrm{SL} 12}$ & $f_{\mathrm{HRC}}^{\mathrm{SL} 12}$ & $f_{\mathrm{Dis}}^{\mathrm{SL} 12}$ & $f_{\text {Merge }}^{\text {C19 }}$ & $\begin{array}{l}f_{\mathrm{GMC}}^{\mathrm{C} 19} \\
\end{array}$ & $f_{\mathrm{HRC}}^{\mathrm{C} 19}$ \\
\hline \multirow{5}{*}{$\overline{0.9}$} & & & 1.10 & 60.0 & 0.33 & 0.00 & 0.51 & 0.14 & 0.35 & 0.33 & 0.47 & 0.20 \\
\hline & & & 1.15 & 52.5 & 0.47 & 0.00 & 0.44 & 0.19 & 0.37 & 0.35 & 0.37 & 0.28 \\
\hline & & & 1.20 & 42.5 & 0.54 & 0.04 & 0.58 & 0.06 & 0.32 & 0.40 & 0.52 & 0.08 \\
\hline & & & 1.20 & 45.0 & 0.54 & 0.00 & 0.42 & 0.16 & 0.42 & 0.41 & 0.39 & 0.20 \\
\hline & & & 1.20 & 52.5 & 0.54 & 0.00 & 0.33 & 0.21 & 0.46 & 0.43 & 0.27 & 0.30 \\
\hline & & & 1.15 & 52.5 & 0.43 & 0.00 & 0.57 & 0.10 & 0.33 & 0.17 & 0.56 & 0.27 \\
\hline & & & 1.15 & 60.0 & 0.43 & 0.00 & 0.53 & 0.15 & 0.32 & 0.15 & 0.52 & 0.33 \\
\hline & & & 1.20 & 45.0 & 0.53 & 0.00 & 0.59 & 0.14 & 0.27 & 0.15 & 0.54 & 0.31 \\
\hline & & & 1.20 & 52.5 & 0.53 & 0.00 & 0.48 & 0.23 & 0.29 & 0.15 & 0.41 & 0.44 \\
\hline & & & 1.20 & 60.0 & 0.53 & 0.00 & 0.48 & 0.27 & 0.25 & 0.08 & 0.44 & 0.48 \\
\hline
\end{tabular}

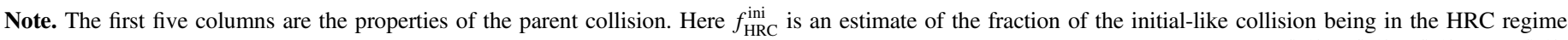

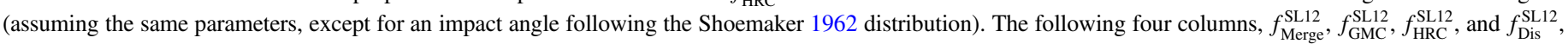

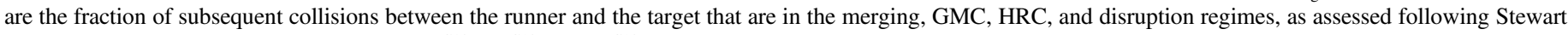

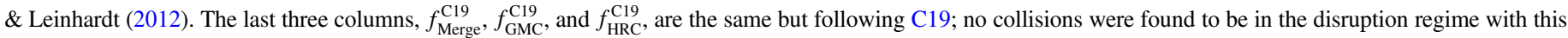
procedure.

with $m_{\text {imp }}=0.5 M_{\oplus}, v_{\text {coll }} / v_{\text {esc }}=1.20$, and $\theta_{\text {coll }}=45^{\circ}$. The main effect is to reduce the overall eccentricity of the remnants, therefore keeping them further away from the other bodies while increasing their potential encounters. We observe two main consequences: the number of collisions between the two remnants is increased from 0.425 to 0.552 , and the return collision velocity is reduced so that the relative difference in the departing velocity is about $10^{-4}$. The other results are in good agreement with the ones discussed previously.

Assuming that the target is on a circular orbit prior to the collision does not lead to a special situation. The deflection occurring during the collision leads to similar eccentricities in both bodies. However, the eccentricities are even lower when the center of mass is assumed to be on a circular orbit, and in this case, the return rate increases while lower return collision velocities are obtained. The differences between the two cases remain limited. The collisions with the larger impactor, $m_{\text {imp }}=0.5 M_{\oplus}$, result in slighter higher eccentricities. This can in part explain the increased rate of collision with other bodies compared to the smaller impactor.

\subsection{Return Collision Type}

We finish the analysis of the HRRs by determining the type of collision they would produce. Direct modeling of each event with SPH would bear a prohibitive computational cost; therefore, we assess the types of those events using scaling laws. We use both the scaling of Stewart \& Leinhardt (2012, hereafter SL12), which is based on Leinhardt \& Stewart (2012), and the modification that in the grazing regime, there is a further division between GMCs and HRCs based on the results from Kokubo \& Genda (2010) and C19. The results of this analysis are provided in Table 4. For comparison, we provide the likelihood for the initial collision to be HRCs, $f_{\mathrm{HRC}}^{\text {ini }}$, by estimating the impact angle for which the transition between GMCs and HRCs occurs for the given bodies and impact velocity using the results from Table 1 and then using the uniform distribution in space following Shoemaker (1962) to asses the probability that the impact angle is greater than that value.
The results using both methods are in good agreement when it comes to the HRC and GMC regimes, with some differences for the fraction of HRCs in the most massive impactor. Quite a number of collisions are found to be close to the transition between GMCs and HRCs; hence, there is some uncertainty associated with the classification performed in C19. For the others, the discrepancy between "merge" and "disruption" is due to the different classification. The disruption regime in SL12 includes partial accretion onto the target, while the merging regime in $\mathrm{C} 19$ also encompasses the part where some material is ejected in the form of debris. These two categories should be seen as collisions that do not produce a second remnant, transient or not.

We obtain that when an HRR occurs, it is more likely to be a merger (direct or GMC) than the previous collision. This relates to the decrease of the relative velocity following the initial HRC. As the HRR impact velocity is lower than in the previous collision, the transition shifts to higher impact angles, therefore reducing the probability of subsequent HRCs. Slowly departing runners close to the mutual escape velocity have a low probability of subsequent HRCs $(<10 \%)$, while for the more rapid ones, this figure increases to roughly $30 \%$. Series of HRC collisions would then mostly end after a few events. This result is consistent with the outcome from Chambers (2013), which found that the accretion timescales of terrestrial planets are increased by a factor on the order of two.

To asses the most favorable situation for a series of HRCs ( i.e., multiple HRRs) between the same bodies, we are interested in the overall fraction of runners that return with an HRC. There are two competing effects: for a runner departing at higher velocity, the proportion of HRRs again being HRCs increases, but at the same time, the fraction of runners that return to the target is reduced. Taking into account both effects, we observe that the overall number of HRC returns increases with the departing velocity, with up to $9 \%$ of the dynamical evolution runs performed in the $m_{\mathrm{imp}}=0.2 M_{\oplus}$ case (for both $v_{\text {coll }} / v_{\text {esc }}=1.15$ with $\theta_{\text {coll }}=60^{\circ} .0$ and $v_{\text {coll }} / v_{\text {esc }}=1.20$ with $\left.\theta_{\text {coll }}=52^{\circ} .5\right)$ and up to $7 \%$ in the $m_{\text {imp }}=0.5 M_{\oplus}$ case. Even though it is unlikely, multiple HRCs between the same bodies 
are possible. This kind of event requires an initial collision that produces a rapidly departing runner, since the relative velocity is expected to decrease at each encounter, ultimately ending in a merging collision. Furthermore, the mass contrast is increased following an $\mathrm{HRC}$, as net mass transfer from the impactor to the target is the norm. Hence, a return collision with more dissimilar-sized bodies and lower velocity reduces the likelihood of grazing events, either GMCs or HRCs. The last effect does not strongly affect the results, as the mass change is relatively small, usually less than $10 \%$ of the impactor's mass, which results in a change of radius of a few percent at most.

As for the more rapidly departing runner, the fraction of collisions with other bodies is comparable with that of the target; another path for multiple HRCs is with intermediate collisions occurring with other bodies.

We discussed in Section 3.3.1 that the presence of additional bodies tends to increase the scatter of the velocity of the return collision. For grazing collisions, the outcome is strongly dependent on the velocity in this range. Thus, the results presented here will also depend on the configuration of the system. However, if the impact angle and velocity are uncorrelated, then the effect is lower, as lower-velocity collisions will tend to favor GMCs, while HRCs are preferred for more energetic events. For instance, if we compare the types obtained with those obtained from the dynamical evolution with no other bodies present, then we find that the types of collisions are quite similar, with a lower number of HRCs in a few series.

In our situation, there is mainly one body with which the collision remnants can closely interact: Venus. With more bodies present, such as what can be expected during the formation of planetary systems, the number of interactions would increase, thereby reducing the relationship of the velocity between successive collisions. If that is the case, the fraction of HRCs is also less constrained, making collision chains more probable.

\section{Discussion and Conclusion}

In this work, we model HRCs that are likely to occur at the end of planetary systems formation (the "late stage") and follow the dynamical evolution of the resulting bodies to see if they recollide. In the following section, we summarize the overall conclusions and discuss the implications of the results on scenarios proposed to explain certain features of the solar system.

\subsection{Return Probability}

Under the gravitational influence of other bodies, the return probability of the runner is heavily reduced. This is particularly true for moderately fast runners $\left(v_{\mathrm{dep}} / v_{\mathrm{esc}} \geqslant 1.1\right)$. When the dynamical evolution is performed without other bodies present - that is, only the Sun, the target, and the runner-the return probability is barely affected by the departing velocity and is always around $90 \%$. But this is an unrealistic scenario. When the other inner planets of the solar system are included during the dynamical evolution (up to and including Saturn), the return probability is greatly affected by the departing velocity. Even for moderate departing velocities on the order of $v_{\text {dep }} / v_{\text {esc }} \simeq 1.1$, the likelihood that the runner collides with a different target is similar to the likelihood that it returns to the same initial target. In summary, the assumption that runners return to the same target body is valid only when there are no other bodies present. When a system of planets is present, direct modeling of the runner trajectory is necessary to obtain a good fidelity of the results.

\subsection{Delay between Successive Collisions}

We find delays between successive collisions on the order of $10^{5}-10^{6} \mathrm{yr}$ for collisions occurring at $1 \mathrm{au}$. We expect the location of the collision to be important, as the delay should be related to the orbital period. For comparison, Chambers (2013) found that treating collisions more realistically, i.e., by adding the possibility of multiple remnants, increases the formation time by a factor of about two. Our delays are thus consistent with these findings, as they are shorter than the time span for the formation of the solar system's terrestrial planets (a few tens of millions of years).

For planets forming during the earlier times of a stellar system, i.e., before the nebula vanishes (around $3 \times 10^{6} \mathrm{yr}$; Haisch et al. 2001), the consequences can be different. In this case, the time span between collisions is comparable to the duration of the stage, except for the close-in planets.

\subsection{Collision Geometry}

The orientation between the orbital planes of the prior and returning collisions is essentially compatible with a uniform distribution. The return collision then has no knowledge of the orientation of the prior impact. If our results are also applicable to any series of giant impacts, then it poses constraints on scenarios that involve multiple collisions or the implications thereof.

Giant impacts are a convenient means to explain planetary rotations, and grazing collisions are found to be useful to explain satellite systems: Earth's Moon (Benz et al. 1986; Canup \& Asphaug 2001; Canup 2004), Mars's Phobos and Deimos (Citron et al. 2015; Hyodo et al. 2017), Pluto-Charon (Canup 2005, 2011), and Haumea (Leinhardt et al. 2010). However, if the orientation of giant impacts is not correlated, then it is unlikely for multiple collisions to happen close to the equatorial plane of the target body and in the prograde direction, first to spin up the body into a rapid rotator and then for the proposed moon-forming collision to take place. This greatly diminishes the likelihood of scenarios that require such a situation (Canup 2008; Cuk \& Stewart 2012).

If the impactors indeed follow a uniform distribution, scenarios that require alignments of multiple collisions, such as in Rufu et al. (2017) and Citron et al. (2018), are also very unlikely. Each collision would contribute to the total angular momentum with a different orientation, and the final value would be random or close to zero, leaving no possibility for a satellite to survive. Hence, if our Moon is the product of one or more collisions, only a small number of those would be able to provide the rotationally oriented material from which the Moon accretes.

\subsection{Type of Return Collision}

Energy dissipation through shocks during the collision leads to a runner departing at a lower velocity compared to the preimpact velocity. The steeper the impact angle, the greater the dissipation. Since the median returning velocity is the same as the departing velocity, the return collisions usually happen at a lower velocity than the initial collision. Combining this with 

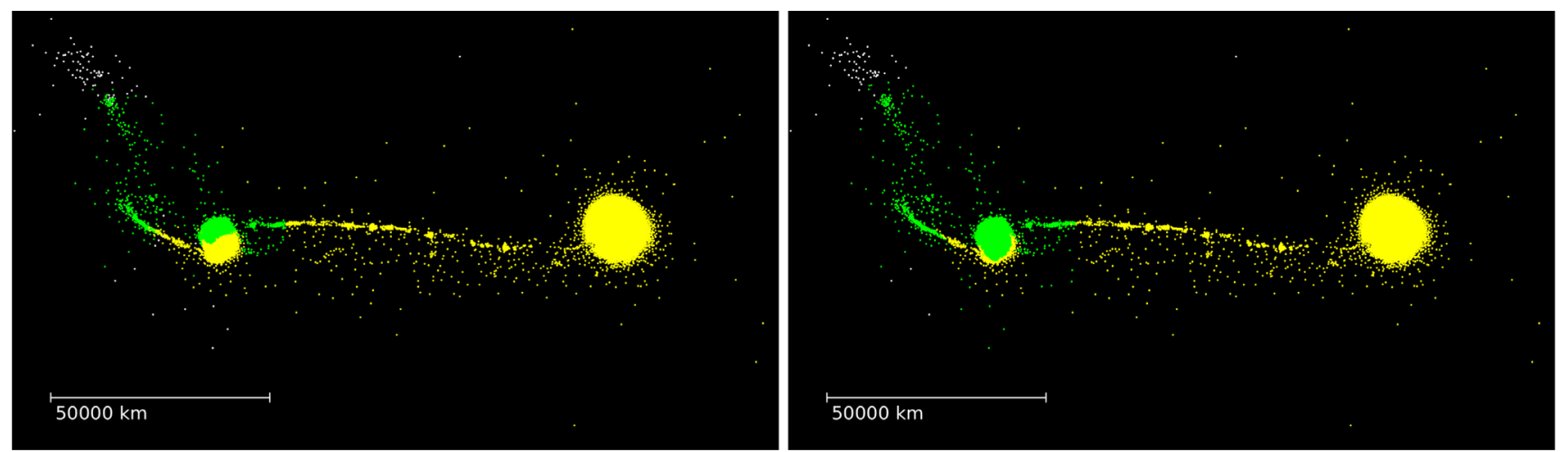

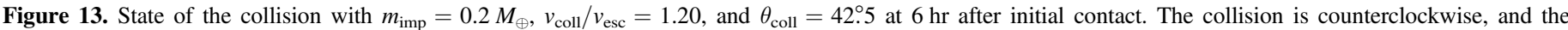

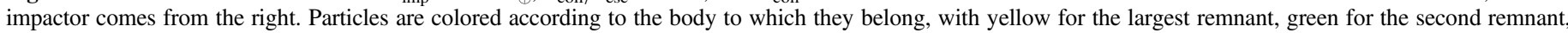

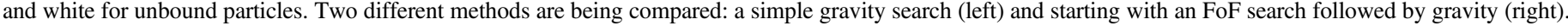

the normal slight mass transfer from the impactor to the target during an HRC, the returning collision has a greater likelihood of being a merger.

A consequence is that a series of low-velocity HRRs between the same objects is unlikely; in order to maintain a sufficiently high impact velocity for the returning collision to also be in the HRC regime, the initial collision must happen at a high velocity. In the latter case, however, the probability of the runner returning to the same body is diminished, leaving an overall low probability for multiple HRRs in series with the same target and a greater probability of scenarios where a higher-energy runner goes from target to target. When considering the ultimate survival of a runner, as proposed by Asphaug \& Reufer (2014) for the origin of Mercury, one must therefore consider a longer-term dynamical evolution to determine whether a runner eventually becomes accreted or survives.

To track the runner with sufficient accuracy in general planetary formation simulations, a sufficiently precise collision model is required. This model must provide not only the masses of the resulting bodies but also their orbital parameters. Furthermore, the outcome for HRCs must be properly returned, as it is quite different from that of a close encounter: relative velocity is reduced, and mass transfer occurs.

\subsection{Material Mixing}

The HRCs are known to be able to alter the bulk composition of bodies, mostly by stripping the outer layer, either silicate (Marcus et al. 2009; and supposedly for the specific case of Mercury) or water ice (Marcus et al. 2010; Burger et al. 2018). Composition changes can produce diverse types of planetesimals, which could potentially explain the specific Earth's element content (Bonsor et al. 2015; Carter et al. 2015).

Collision-induced mixing also has implications in the general context of planetary formation. Relatively head-on HRCs can be a source of significant material mixing, where in typical simulations, about $20 \%$ of the runner's mantle is composed of target material, while the target's mantle bears nearly $10 \%$ of impactor material. Therefore, HRCs are a potential source of equilibration between the planetary bodies. Coupled with a low return probability on the same body, moderately fast runners have the ability to impact other objects afterward, leading to a complex picture of geochemical evolution that can provide a source of equilibration between planetary bodies.

Looking at the collision probabilities from Table 3, we note that a runner from Earth reaching Venus is roughly 10 times more likely than Mars. It is therefore possible that Venus and Earth have equilibrated following an HRC on Earth whose runner later impacted Venus, while Earth and Mars did not. If this was the case, then Venus would show a composition more similar to Earth than expected from standard formation scenarios.

The authors thank Saverio Cambioni, Travis S. J. Gabriel, Alan P. Jackson, and Stephen R. Schwartz for fruitful discussions and an anonymous reviewer for comments and edits that helped improve the clarity of the manuscript. We acknowledge support from NASA grant NNX16AI31G and the University of Arizona. An allocation of computer time from UA Research Computing High Performance Computing (HPC) is gratefully acknowledged.

Software: Mercury (Chambers 1999), matplotlib (Hunter 2007).

\section{Appendix Searching for Bodies}

Searching for remnants in GMCs or HRCs close to the boundary between the two regimes can be tricky. Small fluctuations in velocity are sufficient to result in the bodies being gravitationally bound or not. For runners that are barely escaping or barely bound, the problem is exacerbated by the rotation induced from the encounter. A simple gravitational search, treating all SPH particles independently, fails to grasp this effect and treats parts of the rotating runner in different ways. An example of such behavior is shown in the left panel of Figure 13. It can be seen that a part of the runner, while unbound as a whole, is still found to be part of the largest remnant. The collision happens counterclockwise, and so does the spin of the bodies. Particles whose motion due to body rotation is toward the target (at the bottom of Figure 13) are found to be bound to the target rather than the runner.

Starting the search for bodies with an FoF walk mitigates most of this problem and is the approach we have taken here. For this search, particles that have a density of at least $3 / 4$ of the reference density from the equation of state and are closer than twice their mean smoothing length are deemed to form a single body. For the later gravity search, bodies determined by 

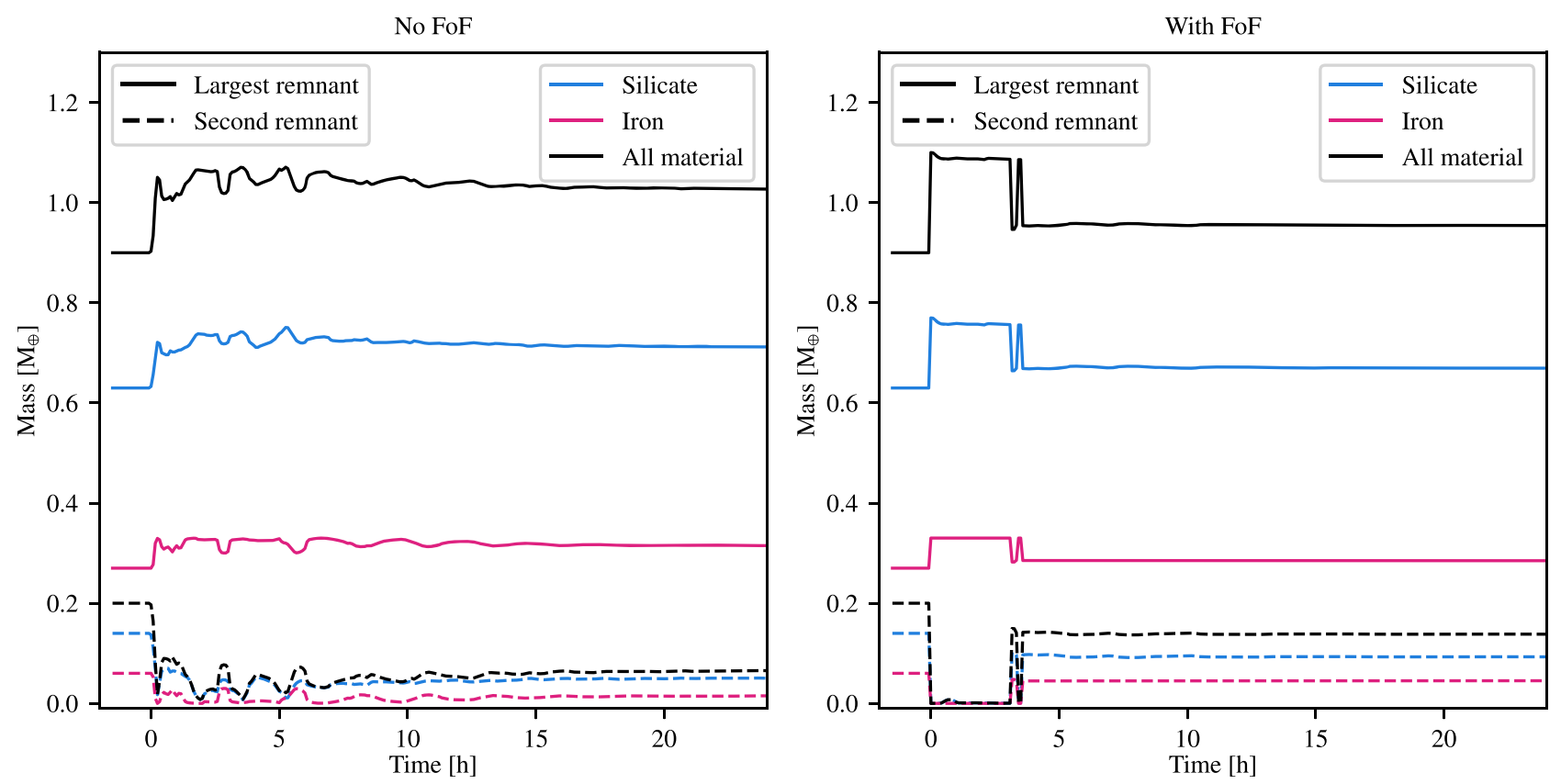

Figure 14. Mass of the clumps vs. time since initial contact for the collision with $m_{\mathrm{imp}}=0.2 M_{\oplus}, v_{\text {coll }} / v_{\mathrm{esc}}=1.20$, and $\theta_{\text {coll }}=42.5$ for two different methods: simple gravity search (left) and starting with an FoF search followed by gravity (right). The values at negative times give the initial state.

the FoF method are treated as a single superparticle. With this scheme, the runner is first determined as a single body and remains as such during the gravity search. Hence, the rigid body rotation is averaged out and a negligible influence on the search algorithm. Only a small number of particles lying on the surface of the runner are not found by the FoF algorithm and are still deemed to be part of the largest remnant, but their contribution to the total mass is small.

The final outcome of the two methods is shown in Figure 14. With a simple gravity search, the clump masses take about $18 \mathrm{hr}$ to converge, while when an FoF search is included, the whole set is initially found to be a single body until the arm that connects them vanishes, and then only small fluctuations continue. But most importantly, the two methods do not converge to the same value; the simple gravity search misses a part of the runner that happens to be rotating toward the target. In addition, since the part of the runner that is found to be part of the largest body has a net motion, other properties, such as the relative velocity, are affected. The converse in a GMC, where part of the transient body is found to not be bound to the largest body, is also possible. Hence, an FoF search is a necessity for outcomes of collisions that are close to the transition between the GMC and HRC regimes.

An FoF search has a further advantage in GMCs. A gravity search is unable to distinguish the transient body, as it is still bound to the target. An FoF is not affected by this and makes us able to compute the orbit of the transient body until later accretion. We will use this feature to more accurately evolve weakly bound GMCs where the body has still not been accreted after $24 \mathrm{hr}$.

\section{ORCID iDs}

Alexandre Emsenhuber (iD https://orcid.org/0000-00028811-1914

Erik Asphaug (iD https://orcid.org/0000-0003-1002-2038

\section{References}

Agnor, C., \& Asphaug, E. 2004, ApJL, 613, L157

Agnor, C. B., Canup, R. M., \& Levison, H. F. 1999, Icar, 142, 219

Alexander, S. G., \& Agnor, C. B. 1998, Icar, 132, 113

Asphaug, E. 2010, ChEG, 70, 199

Asphaug, E. 2017, in Planetesimals: Early Differentiation and Consequences for Planets, ed. L. T. Elkins-Tanton \& B. P. Weiss (Cambridge: Cambridge Univ. Press), 737

Asphaug, E., Agnor, C. B., \& Williams, Q. 2006, Natur, 439, 155

Asphaug, E., \& Reufer, A. 2013, Icar, 223, 544

Asphaug, E., \& Reufer, A. 2014, NatGe, 7, 564

Barnes, J., \& Hut, P. 1986, Natur, 324, 446

Benz, W., Anic, A., Horner, J., \& Whitby, J. A. 2007, SSRv, 132, 189

Benz, W., Slattery, W. L., \& Cameron, A. G. W. 1986, Icar, 66, 515

Benz, W., Slattery, W. L., \& Cameron, A. G. W. 1988, Icar, 74, 516

Bonsor, A., Leinhardt, Z. M., Carter, P. J., et al. 2015, Icar, 247, 291

Burger, C., Maindl, T. I., \& Schäfer, C. M. 2018, CeMDA, 130, 2

Cambioni, S., Asphaug, E., Emsenhuber, A., et al. 2019, ApJ, 875, 40

Canup, R. M. 2004, Icar, 168, 433

Canup, R. M. 2005, Sci, 307, 546

Canup, R. M. 2008, Icar, 196, 518

Canup, R. M. 2011, AJ, 141, 35

Canup, R. M., \& Asphaug, E. 2001, Natur, 412, 708

Carter, P. J., Leinhardt, Z. M., Elliott, T., Walter, M. J., \& Stewart, S. T. 2015, ApJ, 813, 72

Chambers, J. E. 1999, MNRAS, 304, 793

Chambers, J. E. 2001, Icar, 152, 205

Chambers, J. E. 2006, Icar, 180, 496

Chambers, J. E. 2013, Icar, 224, 43

Chandrasekhar, S. 1969, Ellipsoidal Figures of Equilibrium (New Haven, CT: Yale Univ. Press)

Chau, A., Reinhardt, C., Helled, R., \& Stadel, J. 2018, ApJ, 865, 35

Citron, R. I., Genda, H., \& Ida, S. 2015, Icar, 252, 334

Citron, R. I., Perets, H. B., \& Aharonson, O. 2018, ApJ, 862, 5

Ćuk, M., \& Stewart, S. T. 2012, Sci, 338, 1047

Emsenhuber, A. 2017, PhD thesis, Univ. Bern

Emsenhuber, A., Jutzi, M., \& Benz, W. 2018, Icar, 301, 247

Gabriel, T. S. J., Jackson, A. P., Asphaug, E., et al. 2019, ApJ, submitted

Haisch, K. E., Lada, E. A., \& Lada, C. J. 2001, ApJL, 553, L153

Hunter, J. D. 2007, CSE, 9, 90

Hyodo, R., Genda, H., Charnoz, S., \& Rosenblatt, P. 2017, ApJ, 845, 125

Ida, S., \& Lin, D. N. C. 2004, ApJ, 604, 388

Inaba, S., Tanaka, H., Nakazawa, K., Wetherill, G. W., \& Kokubo, E. 2001, Icar, 149, 235 
Inamdar, N. K., \& Schlichting, H. E. 2016, ApJL, 817, L13

Jackson, A. P., Gabriel, T. S. J., \& Asphaug, E. I. 2018, MNRAS, 474, 2924

Jackson, A. P., \& Wyatt, M. C. 2012, MNRAS, 425, 657

Kokubo, E., \& Genda, H. 2010, ApJL, 714, L21

Kokubo, E., \& Ida, S. 2002, ApJ, 581, 666

Leinhardt, Z. M., Dobinson, J., Carter, P. J., \& Lines, S. 2015, ApJ, 806, 23

Leinhardt, Z. M., Marcus, R. A., \& Stewart, S. T. 2010, ApJ, 714, 1789

Leinhardt, Z. M., \& Stewart, S. T. 2012, ApJ, 745, 79

Leleu, A., Jutzi, M., \& Rubin, M. 2018, NatAs, 2, 555

Marcus, R. A., Sasselov, D., Stewart, S. T., \& Hernquist, L. 2010, ApJL, 719, L45

Marcus, R. A., Stewart, S. T., Sasselov, D., \& Hernquist, L. 2009, ApJL, 700, L118

Melosh, H. J. 2007, M\&PS, 42, 2079

Monaghan, J. J. 1992, ARA\&A, 30, 543
Raymond, S. N., O'Brien, D. P., Morbidelli, A., \& Kaib, N. A. 2009, Icar, 203, 644

Reufer, A., Meier, M. M. M., Benz, W., \& Wieler, R. 2012, Icar, 221, 296

Rosswog, S. 2009, NewAR, 53, 78104

Rufu, R., Aharonson, O., \& Perets, H. B. 2017, NatGe, 10, 89

Schlichting, H. E., Sari, R., \& Yalinewich, A. 2015, Icar, 247, 81

Shoemaker, E. M. 1962, in Physics and Astronomy of the Moon, ed. Z. Kopal (New York: Academic), 283

Stewart, S. T., \& Leinhardt, Z. M. 2012, ApJ, 751, 32

Thommes, E. W., Duncan, M. J., \& Levison, H. F. 2003, Icar, 161, 431

Thompson, S. L., \& Lauson, H. S. 1972, Improvements in the CHART-D Radiation-hydrodynamic code III: Revised analytic equations of state, Tech. Rep. SC-RR-71 0714, Sandia National Laboratories

Wetherill, G. W. 1985, Sci, 228, 877 